\title{
The Adjustment of Barotropic Currents at the Shelf Break to a Sharp Bend in the Shelf Topography
}

\author{
by \\ William James Williams \\ B.A. University of Cambridge, \\ (1991) \\ Submitted in partial fulfillment of the \\ requirements for the degree of \\ MASTER OF SCIENCE \\ at the
MASSACHUSETTS INSTITUTE OF TECHNOLOGY
and the \\ WOODS HOLE OCEANOGRAPHIC INSTITUTION \\ February 1996 \\ (C) William James Williams 1995
}

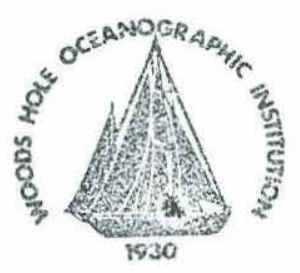

The author hereby grants to MIT and to WHOI permission to reproduce and to distribute copies of this thesis document in whole or in part.

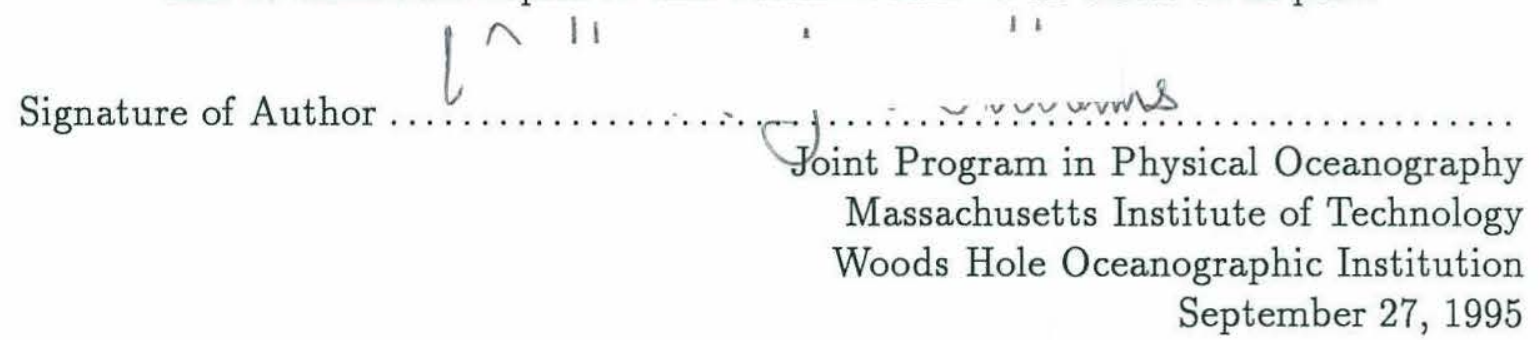

Certified by

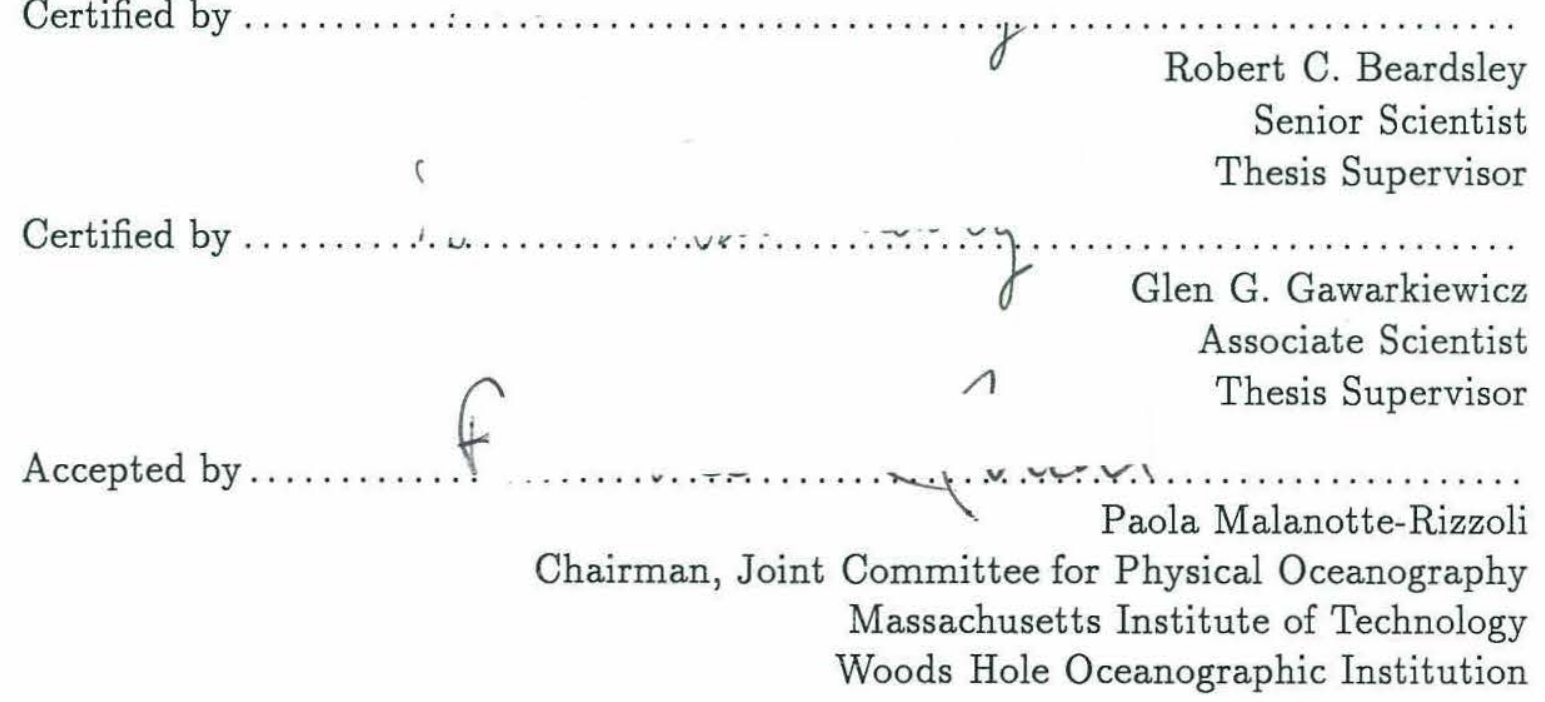




\title{
The Adjustment of Barotropic Currents at the Shelf Break to a Sharp Bend in the Shelf Topography
}

\author{
by \\ William James Williams
}

\begin{abstract}
Submitted to the Massachusetts Institute of Technology/Woods Hole Oceanographic Institution Joint Program in Oceanography/Applied Ocean Science and Engineering in partial fulfillment of the requirements for the degree of Master of Science in Oceanography
\end{abstract}

\begin{abstract}
During January-March, Scotian Shelf water has been observed to flow episodically from the southwestern Scotian Shelf directly across the Northeast Channel to Georges Bank. The possible factors that allow Scotian Shelf water to break the topographic constraint presented by the Northeast Channel and flow directly to Georges Bank are considered. As a simple analog to the flow over the southwestern Scotian Shelf near the Northeast Channel, the adjustment of a barotropic current near a shelf-break to a sharp bend in the shelf topography is studied numerically. For parameters within the oceanographic range, the adjustment to the bend is smooth and steady with no eddies shed at the corner. The vorticity dynamics allow a balance between the vortex stretching in the flow and the curvature in the flow. This is possible since the bend is a right-hand one facing downstream, a similar balance not being possible for a left-hand bend, in which case eddy formation is likely. A simple model of this balance clarifies the vorticity dynamics and provides the scaling $r_{c}=\sqrt{\epsilon} L / 0.765$ for any streamline in the flow, where $r_{c}$ is the radius of curvature at the corner, $\epsilon=u_{0} / f L$ and $L=h_{0} / b$, where $u_{0}$ is the initial speed, $f$ the coriolis parameter, $h_{0}$ the initial depth and $b$ the bottom slope. These results show that other factors such as stratification, wind stress, and time-dependent inflow must play a role in any flow across the Northeast Channel.
\end{abstract}

Thesis Supervisor: Dr. Robert C. Beardsley

Title: Senior Scientist, Physical Oceanography, Woods Hole Oceanographic Institution

Thesis Supervisor: Dr. Glen G. Gawarkiewicz

Title: Associate Scientist, Physical Oceanography, Woods Hole Oceanographic Institution 


\section{Acknowledgements}

I wish to thank Bob Beardsley and Glen Gawarkiewicz, my thesis advisors, for many helpful discussions on various aspects of the science, numerical modeling, and writing of this thesis.

Thanks also go to my committee members, Glenn Flierl, Paola MalanotteRizzoli and Dave Chapman, for their time and help and to Jamie Pringle for advice on numerical modeling, UNIX and Fortran.

I am very grateful to the US-GLOBEC program for providing the funding for this study (N.S.F. grant OCE-9313671). This is GLOBEC contribution number 37. 


\section{Contents}

$\begin{array}{ll}\text { Abstract } & 3\end{array}$

Acknowledgements $\quad 5$

1 Introduction $\quad 9$

1.1 Northeast Channel Area . . . . . . . . . . . . . . . . . 9 9

1.2 The Model . . . . . . . . . . . . . . . . . . 12

2 The Numerical Model $\quad 15$

2.1 Introduction . . . . . . . . . . . . . . . . . . . 15

2.2 Vertical Structure . . . . . . . . . . . . . . . . . . . . . 17

2.3 The Numerical Solution Technique . . . . . . . . . . . . . . . 18

2.4 Numerical Resolution and Stability . . . . . . . . . . . . . . . . . . . 19

2.5 Open Boundary Conditions . . . . . . . . . . . . . . 21

2.5.1 No-gradient open boundary condition. . . . . . . . . . 21

2.5.2 Orlanski open boundary condition . . . . . . . . . . . . 22

3 The Base Case $\quad 25$

3.1 Introduction . . . . . . . . . . . . . . . 25

3.2 The Model Configuration . . . . . . . . . . . . . . . . . 26

3.3 Description of the Flow . . . . . . . . . . . . . . . . . . . . . . . . . . . . 28

3.4 Vorticity Dynamics . . . . . . . . . . . . . . . . . . 36

3.5 A Simple Model . . . . . . . . . . . . . . . . . . . . 39

4 Scalings and Parameter Variations 43

4.1 Introduction . . . . . . . . . . . . . . . . . . . . . . . . . . . . . . . . . . .

4.2 Inflow Velocity . . . . . . . . . . . . . . . . . 44

4.3 Scaling . . . . . . . . . . . . . . . . . . . . . . . . . . . . . . . . . . . 44

4.4 Other Variations . . . . . . . . . . . . . . . . . . . . . . 49

4.5 Different Topographies .................... 52 
5 Conclusions $\quad 53$

5.1 Discussion and Conclusions ................... 53

$\begin{array}{ll}\text { Appendix A } & 55\end{array}$

A Stability of a Barotropic Gaussian Jet Over Sloping Bathymetry 55

$\begin{array}{ll}\text { Bibliography } & 57\end{array}$ 


\section{Chapter 1}

\section{Introduction}

\subsection{Northeast Channel Area}

The mean flow over the Scotian Shelf and the Mid-Atlantic Bight is equatorward. Chapman et. al. (1986) show that this equatorward flow over the Mid-Atlantic Bight can be considered a downstream extension of the flow over the Scotian Shelf. However, the Gulf of Maine and Georges Bank lie between the Scotian Shelf and the Mid-Atlantic Bight and the circulation is complex due to a possible bifurcation of the flow at the equatorward end of the Scotian Shelf where the Northeast Channel separates the southwestern Scotian Shelf from Georges Bank (Figure 1.1).The mean currents near the shelf-break of the southwestern Scotian Shelf flow southwards to the Northeast Channel and then follow isobaths and turn right into the Gulf of Maine (Smith and Petrie, 1982, Smith, 1983). This flow is rotationally dominated and the wall of the Northeast Channel is viewed as a topographic constraint, preventing the Scotian Shelf water from continuing southward. The purpose of this study is to begin to identify the processes involved with the episodic occurrence of Scotian Shelf water on Georges Bank during late-winter/early-spring. Bisagni et. al. (submitted) show 


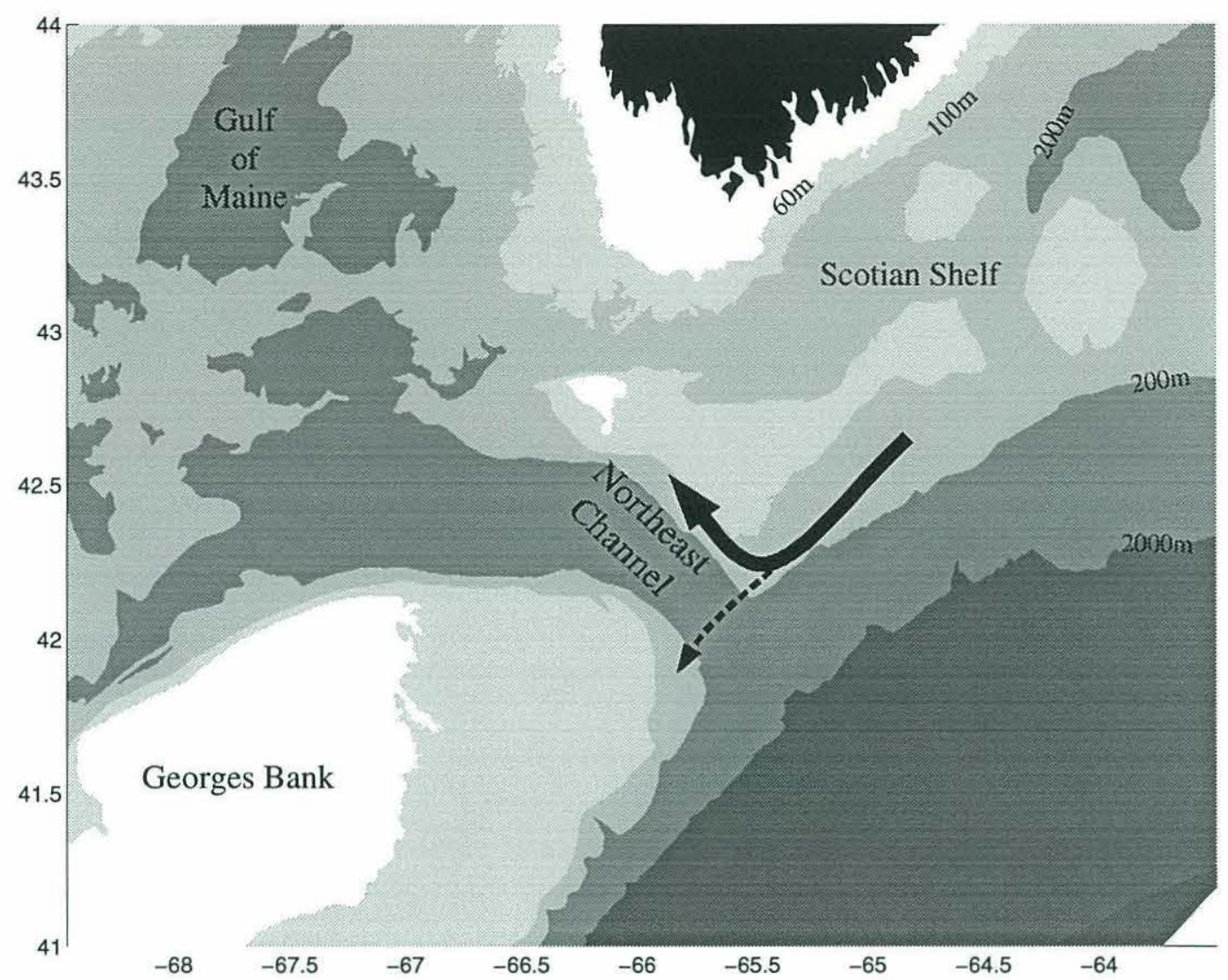

Figure 1.1: A map of the Northeast Channel area showing the mean circulation (solid arrow), and the episodic flow across the Northeast Channel (dashed arrow). The $x$ and $y$ axes are marked in degrees longitude and degrees latitude, respectively.

that during this period Scotian Shelf water can move directly from the southwestern tip of the Scotian Shelf across the Northeast Channel and onto the southern flank of Georges Bank. Inertia, stratification, bottom friction, and wind stress are likely to be involved with this topographic constraint being broken and the Scotian Shelf water crossing the Northeast Channel.

The southward flow over the Scotian Shelf towards the Northeast Channel has a component which flows close to the shelf break (Smith and Petrie, 1982, Smith 1983). Following this current, the shelf-break turns $90^{\circ}$ to the right at the Northeast Channel with a radius of curvature of approximately $6 \mathrm{~km}$. The topographic constraint that 
(a)

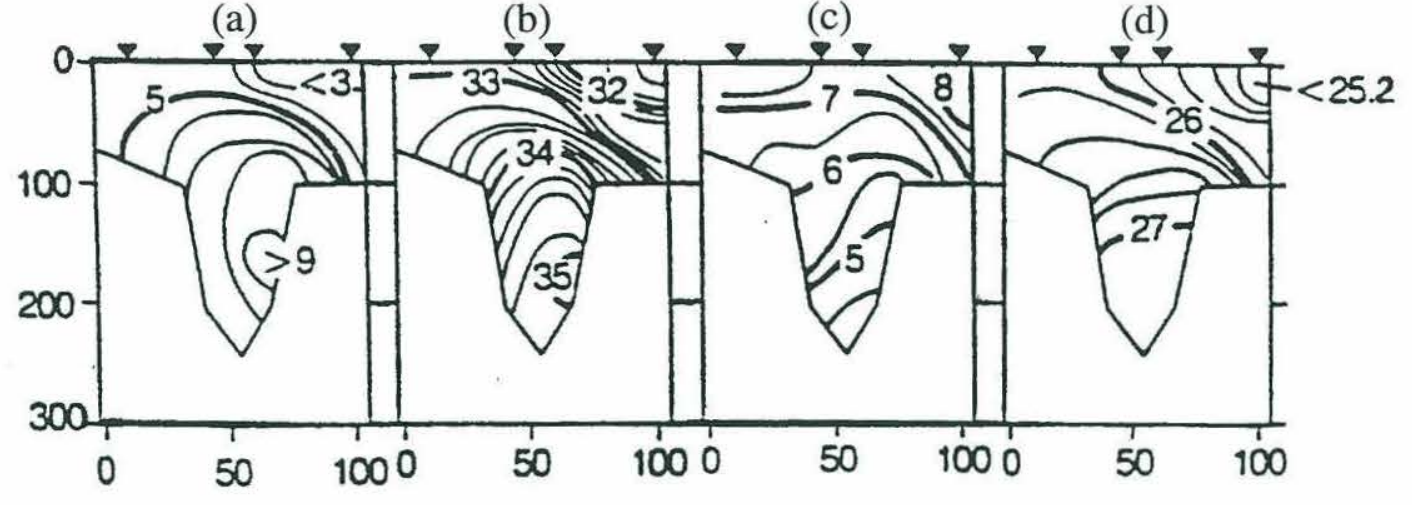

Figure 1.2: A typical section across the Northeast Channel during January-March (Flagg, 1987). The properties shown are: (a) temperature ${ }^{\circ} \mathrm{C},(\mathrm{b})$ salinity ppt, (c) oxygen $\mathrm{mL} / \mathrm{L}(\mathrm{d})$ density $\sigma_{t}$. The Scotian Shelf is on the right and Georges Bank on the left.

barotropic, inviscid, geostrophic flow should follow contours of $f / h$ is weakened at the corner since, at such small scales of flow, the local Rossby number can become large and the non-linear terms in the momentum equation become important. Therefore, due to its inertia, it may be possible for the flow to leave the bathymetry at the corner and begin to cross the Northeast Channel.

Figure 1.2 shows the typical water properties of a cross-section of the Northeast Channel during January-March. The bottom of the Northeast Channel contains warm, salty, and dense oceanic water that is flowing into the Gulf of Maine while the surface water contains fresher, colder, and lighter shelf water flowing into the Gulf of Maine over the Scotian Shelf and out of the Gulf of Maine over Georges Bank. The vertical stratification weakens the topographic constraint of the Northeast Channel by effectively splitting the flow into two layers, thereby reducing the vorticity stretching involved with any cross channel flow in the upper-layer. The inertia of the flow over the Scotian Shelf may then be large enough to carry the Scotian Shelf water to Georges Bank. This stratification is present in all seasons, reaching a maximum in summer, and so if it is an important factor in this problem Scotian Shelf water may cross the Northeast Channel in all seasons if the appropriate forcing is present. However, the 
difference in the water properties between the Scotian Shelf and Georges Bank are relatively small from June through December, and so it may be difficult to identify Scotian Shelf water over Georges Bank for much of the year.

The difference in water properties between Georges Bank and the Scotian Shelf is greatest during January-March. The density front between the two water masses runs diagonally from the sea-floor near the shelf-break of the Scotian Shelf to the sea surface near the middle of the Northeast Channel, with the Scotian Shelf water being more buoyant than the Georges Bank water (see Figure 1.2). Any cross channel flow of Scotian Shelf water will be two layer in nature and involve vorticity stretching between the layers of Scotian Shelf water and Georges Bank water. This may allow Scotian Shelf water to cross the Northeast Channel in winter.

The surface stress due to winds and the bottom stress due to bottom friction may also play a role in forcing the Scotian Shelf water across the Northeast Channel. The wind-stress over the southwestern Scotian Shelf is dominated by the passage of storms during January-March, which provides periods of intense wind forcing. The mean wind-stress during that time of year is directed southeastward (Bisagni et.al, submitted), so the Ekman transport associated with it is directed southwestward, across the Northeast Channel. For the flows over the Scotian Shelf near the Northeast Channel, the Ekman transport associated with bottom friction is directed off the shelf (Gawarkiewicz and Chapman, 1991), and so also tends to force Scotian Shelf water across the Northeast Channel.

\subsection{The Model}

Although it is expected that stratification is important to this problem, it is necessary to study the barotropic case first in order to understand the simplest case involving flow around a curving shelf break. The simplicity of the barotropic case may also lend insight into the more complicated dynamics of the baroclinic case. The problem 


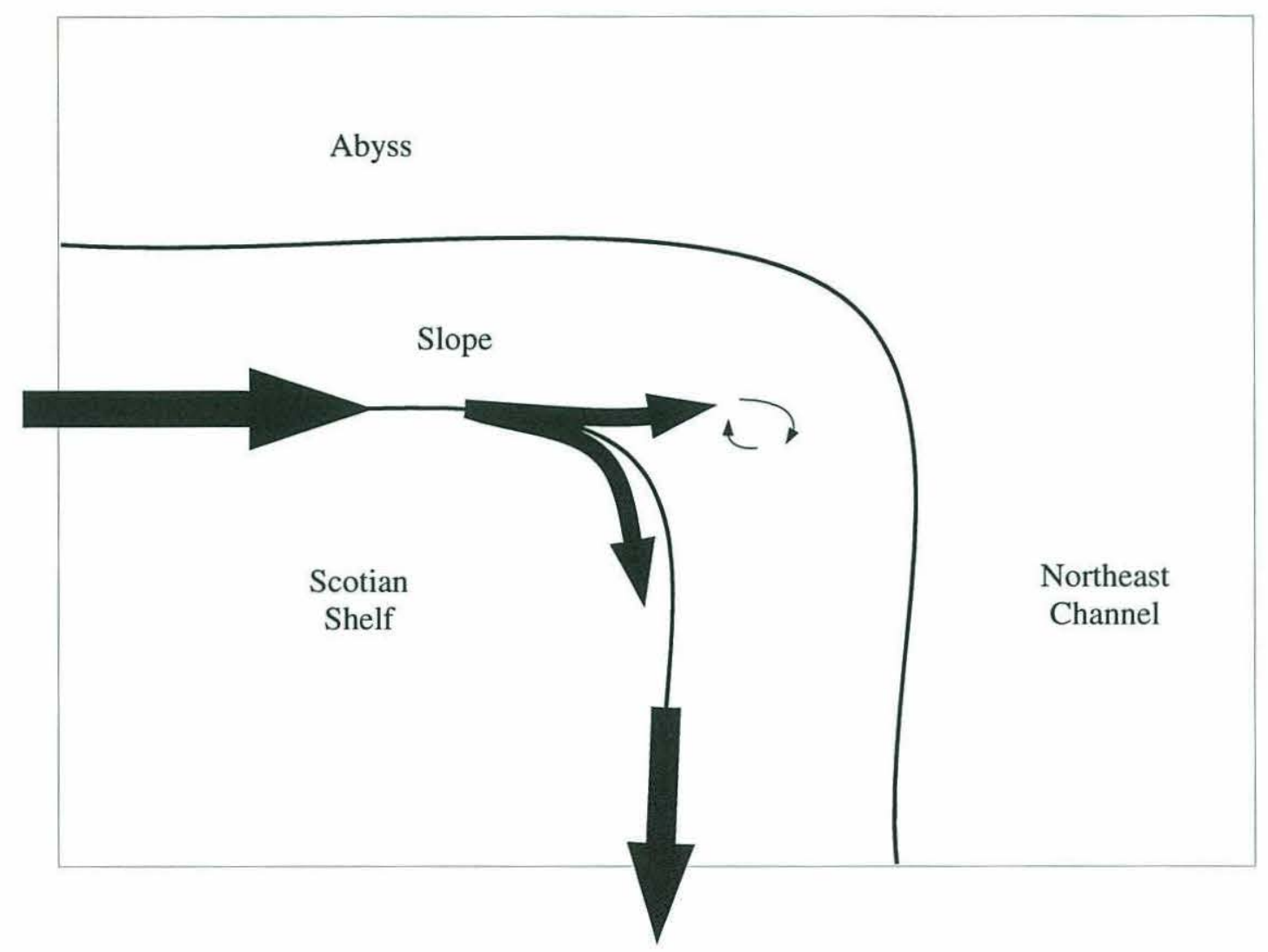

Figure 1.3: A schematic showing model topography representative of the southwestern Scotian Shelf. The arrows represent the flow near the shelf-break and its possible adjustment at the corner.

here is further simplified by considering only the initial separation of the shelf-break current from the Scotian Shelf. The baroclinic Rossby radius for the region is about $15 \mathrm{~km}$ and the width of the Northeast Channel is about $40 \mathrm{~km}$, and so it is further assumed that the dynamics of separation from the Scotian Shelf do not depend on the presence of Georges Bank. This assumption is consistent with the nature of the barotropic adjustment found in this study.

A schematic of the model considered here is shown in Figure 1.3. Only the Scotian Shelf and the $90^{\circ}$ bend in the shelf-break have been retained in the model. The adjustment of barotropic flows near the shelf-break to this bend were studied. 
Klinger (1994) suggested that the appropriate criterion necessary for the separation of a barotropic current from a coast is that the radius of curvature of the coastline should be less than the inertial radius $u / f$ of the flow. When this criterion is met, the flow overshoots the corner and then re-attaches to the coast further downstream, creating a region of recirculation at the coast in which the local Rossby number is greater than one. It was expected that a similar criterion would apply to a current flowing around a bend in the shelf-break, and that if the inertial radius and Rossby number of the flow were large enough, the flow would overshoot the bend and eddies would form. This is found not to be the case for oceanographically reasonable parameters. The flow adjusts to the presence of the corner in a smooth, steady manner and does not form eddies.

A three-dimensional primitive equation model was used to study the adjustment of shelf-break currents to the bend in the shelf-break. This approach was used because the problem did not appear to be tractable analytically and the threedimensional primitive equation model allows the future introduction of baroclinicity, wind-stress, time-dependent forcing, and the resolution of the top and bottom boundary layers.

The model is described in Chapter 2 and a base case examined in Chapter 3. Parameter variations from the base case are studied in Chapter 4 and a simple scaling for the deflection of the flow at the corner is presented. A discussion of the results and conclusions appears in Chapter 5. 


\section{Chapter 2}

\section{The Numerical Model}

\section{$2.1 \quad$ Introduction}

The numerical model used for this study was version 3.0 of the Semi-spectral Primitive Equation ocean Model (SPEM) described by Haidvogel et. al. (1991). SPEM attempts to solve the primitive equations of motion for a Boussinesq, hydrostatic, rotating, fluid. The momentum equations are

$$
\begin{aligned}
\frac{\partial u}{\partial t}+(\mathbf{u} \cdot \nabla) u-f v & =-\frac{1}{\rho_{0}} \frac{\partial P}{\partial x}+F^{x}+D^{x}, \\
\frac{\partial v}{\partial t}+(\mathbf{u} \cdot \nabla) v+f u & =-\frac{1}{\rho_{0}} \frac{\partial P}{\partial y}+F^{y}+D^{y}, \\
\frac{\partial P}{\partial z} & =-\rho g,
\end{aligned}
$$

with the continuity equation

$$
\frac{\partial u}{\partial x}+\frac{\partial v}{\partial y}+\frac{\partial w}{\partial z}=0,
$$

where $(u, v, w)$ is the velocity in the $(x, y, z)$ direction, $P$ is the pressure, $f$ the Coriolis parameter, $\rho_{0}$ the reference density, $\rho$ the total density, $g$ the acceleration due to 
gravity, $\left(F^{x}, F^{y}\right)$ the forcing term in the $(x, y)$ direction, and $\left(D^{x}, D^{y}\right)$ the dissipative term in the $(x, y)$ direction. For this study $\left(F^{x}, F^{y}\right)$ are set equal to zero and $\left(D^{x}, D^{y}\right)$ are Laplacian friction of the form

$$
\begin{aligned}
D^{x} & =A_{h}\left(\frac{\partial^{2} u}{\partial x^{2}}+\frac{\partial^{2} u}{\partial y^{2}}\right)+A_{v} \frac{\partial^{2} u}{\partial z^{2}} \\
D^{y} & =A_{h}\left(\frac{\partial^{2} v}{\partial x^{2}}+\frac{\partial^{2} v}{\partial y^{2}}\right)+A_{v} \frac{\partial^{2} v}{\partial z^{2}}
\end{aligned}
$$

where $A_{h}$ is the horizontal viscosity, introduced to damp numerical noise, and $A_{v}$ is the vertical viscosity, introduced to represent the vertical mixing of momentum.

SPEM has a rigid lid. The rigid lid can support a barotropic pressure gradient and so mimic the barotropic pressure gradients which are normally produced by gradients in the free surface elevation. However, with no free surface elevation, there is no mass storage associated with the free surface and wave-like motions which depend on it are not present. These waves (e.g., barotropic tides, surface gravity waves, and barotropic Kelvin waves) are not relevant to this study, the pertinent dynamics being that of vortex stretching due to flow over topography. Thus the presence of the rigid lid (i.e., lack of free surface) does not affect the results of this study.

The pressure $P$ can be decomposed into the surface pressure $P^{s}$ on the rigid lid, and a hydrostatic pressure $P^{h}$

$$
P=P^{s}+P^{h}
$$

The surface pressure is a diagnostic variable in SPEM. SPEM calculates the depthaveraged flow field $(\bar{u}, \bar{v})$ by inverting a depth-averaged vorticity equation for the transport stream function $\Psi$, and so does not require information about the depthindependent forcing from surface pressure gradients. The surface pressure gradient is inferred from the momentum equations (2.1) and (2.2). 
The calculation of the hydrostatic pressure requires knowledge of the density field $\rho$. The density is calculated by solving the advection/diffusion equations for salt and heat and then using an equation of state to calculate density

$$
\begin{aligned}
\frac{\partial T}{\partial t}+(\mathbf{u} \cdot \nabla) T & =F^{T}+D^{T} \\
\frac{\partial S}{\partial t}+(\mathbf{u} \cdot \nabla) S & =F^{S}+D^{S} \\
\rho & =\rho(T, S, P),
\end{aligned}
$$

where $T$ is temperature, $S$ is salt, $F^{T}$ is the temperature forcing, $F^{S}$ is the salt forcing, $D^{T}$ is the temperature diffusion, and $D^{S}$ is the salt diffusion. For this study, the model is run with a constant density $\rho_{0}$ of $1000 \mathrm{~kg} \mathrm{~m}^{-3}$ throughout the domain. There is no flux of heat or salt through the boundaries of the domain so the density remains constant and the flow is barotropic. The advection/diffusion equations for heat and salt are switched off.

\subsection{Vertical Structure}

The governing equations are transformed to sigma-coordinates before attempting to solve them. The transformation between $\sigma$ and $z$ is given by

$$
\sigma=1+2 \frac{z}{h}
$$

so that the model bottom is at $\sigma=-1$ and the model top is at $\sigma=1$. The grid points in the vertical are chosen at fixed sigma values so the vertical grid is stretched in deep regions and compressed in shallow regions.

The solution in the vertical is computed spectrally by representing the vertical structure of any variable as a sum of Chebyshev polynomials. The number of Chebyshev polynomials used in this sum will set the vertical resolution of the model. The grid points in the vertical are chosen so as to lie on the extrema of the highest order 
Chebyshev polynomial used. This allows lower resolution at mid-depth and higher resolution in the boundary layers near the model top and bottom boundaries.

The spectral expansion of a variable is calculated from its values at the vertical grid points. For the purposes of SPEM, this expansion is then used to do vertical integration and differentiation of the model fields.

\subsection{The Numerical Solution Technique}

The horizontal transport is non-divergent because SPEM has a rigid lid. This allows the definition of the transport streamfunction to be

$$
\bar{u}=-\frac{1}{h} \frac{\partial \Psi}{\partial y} \quad, \quad \bar{v}=\frac{1}{h} \frac{\partial \Psi}{\partial x} .
$$

The non-divergence of the horizontal transport can then be guaranteed by solving for the transport streamfunction first and then deducing the horizontal transport from it. The transport streamfunction is obtained from the depth-averaged vorticity by solving the following elliptic equation, given the values of $\Psi$ on the boundaries of the domain

$$
\frac{\partial}{\partial x}\left(\frac{1}{h} \frac{\partial \Psi}{\partial x}\right)-\frac{\partial}{\partial y}\left(\frac{1}{h} \frac{\partial \Psi}{\partial y}\right)=\omega,
$$

where $\omega$ is the depth-averaged vorticity, and $\mathrm{h}$ is the depth.

The flow field is integrated in time in the following manner. The horizontal velocity field is stepped forward using all the forcing except the surface pressure gradient. The model fields are now correct except for their depth-averaged component. The depth-averaged component is then found by stepping forward the depth-averaged vorticity equation, and solving the above elliptic equation for the transport streamfunction and then deducing the depth-averaged flow. The horizontal velocities $u$ and $v$ are therefore prognostic variables and the vertical velocity $w$ is a diagnostic variable computed from the $u$ and $v$ fields through the continuity equation (2.4). 
The top and bottom boundary conditions for $w$ specify no flow through the rigid lid and the model bottom

$$
\begin{aligned}
w & =0 \quad \text { at } \quad z=0, \\
u h_{x}+v h_{y}=0 & \text { at } \quad z=-h .
\end{aligned}
$$

For this study, there is no wind stress so the surface stress boundary condition reduces to

$$
u_{z}=0, \quad v_{z}=0 \text { at } z=0
$$

and at the bottom, the bottom stress is specified though a linear bottom friction parameterization

$$
A_{v} u_{z}=r_{f} u, A_{v} v_{z}=r_{f} v \text { at } z=-h
$$

where $r_{f}$ is the linear drag coefficient.

\subsection{Numerical Resolution and Stability}

SPEM uses a leapfrog time stepping scheme which computes the fields at the current time step using the fields two time steps previously. This causes the fields at the current time step to diverge from the fields at the previous time step. Every fourth time step is a trapezoidal time step which corrects for this 'splitting' of the model fields by, in effect, averaging the fields in time (Mesinger and Arakawa, 1976).

The numerical noise inherent in any finite difference scheme occurs on the scale of $2 \Delta x$, where $\Delta x$ is the resolution of the grid. Laplacian friction in the horizontal with small viscosity was added to the momentum equations to damp out this noise. The viscosity acts to preferentially damp the small scales in the flow and also leads to the broadening of the inflow jet as it flows through the domain. The viscosity was 
then chosen to be small enough $\left(4-100 \mathrm{~m}^{2} \mathrm{~s}^{-1}\right)$ so that the spreading of the jet was negligible.

The horizontal grid size for this model was chosen to be close to $2 \mathrm{~km}$. Features in the flow and topography that are on the order of $10 \mathrm{~km}$ are properly resolved. The smallest non-zero radius of curvature used in this study for the bend in the bathymetry was $10 \mathrm{~km}$ and it was assumed that the smallest scales of the flow fields would also be about this size. It was impossible to resolve the sharp corner in the runs where the radius of curvature of the corner was set equal to zero, so for these runs the horizontal resolution was kept at $2 \mathrm{~km}$. The smallest scales in the flow field were well resolved in these runs.

The time step was chosen so that the advection, wave-propagation, and diffusion contained in (2.1), (2.2), (2.3) were properly resolved. The CFL-like conditions for this are

$$
\begin{aligned}
\frac{\Delta x}{\Delta t} & <u_{\max } \\
\frac{\Delta x}{\Delta t} & <c_{\max }+u_{\max } \\
\frac{\Delta x^{2}}{\nu \Delta t} & >\frac{1}{2}
\end{aligned}
$$

where $u_{\max }$ is the maximum flow speed, $c_{\max }$ is the maximum of the group velocity and phase speed for the waves in the system, and $\nu$ is the horizontal viscosity chosen for the model.

The resolution in the vertical needs to be chosen so that the top and bottom boundary layers are well resolved. Nine vertical modes were used so that the bottom Ekman layer was well resolved over the shelf and the top of the slope when the linear bottom friction was switched on (Gawarkiewicz and Chapman, 1995). Condition (2.18) must also hold in the vertical where $\Delta x$ is the closest spacing of the vertical grid points and $\nu$ is now the vertical viscosity in the model. 
To check that the flow was being properly resolved by the model, the base case was re-run at twice the horizontal resolution and a quarter of the time step so as to maintain condition (2.18). The flow fields produced are essentially identical.

\subsection{Open Boundary Conditions}

The purpose of the open boundaries of the model domain is to allow flow to enter or leave the domain with the same velocity and density that it would have if the domain was unbounded. The flow field cannot be calculated at the open boundary and is not prescribed there, so it must be appropriately estimated from the flow field near the boundary and its dynamical balance. The no-gradient open boundary condition and the Orlanski open boundary condition were both used in this study. These two boundary conditions and the types of flow they were useful for are described below.

\subsubsection{No-gradient open boundary condition.}

This open boundary condition gives no gradient in all the flow fields across the open boundary, i.e.,

$$
\phi_{B}^{t}=\phi_{B-1}^{t}
$$

where $\phi$ represents any variable, $t$ is the current time level and $B$ is the value of the cross-boundary index on the open boundary.

This condition allows the value of the variable at the open boundary to vary with time but allows no variation of the variable across the boundary. This gives an infinite phase speed at the open boundary to wave-like features crossing the open boundary. The no-gradient open boundary condition is then not well suited to allowing strongly time-varying and wave-like flows to pass out of the domain and a large amount of the incident wave energy will be reflected (Chapman, 1985). The 
no-gradient open boundary condition is best suited to allowing steady or slowly timevarying flows to pass through the open boundary.

The domain used for this study has an open boundary that allows the shelf current to leave the domain after it has encountered the corner in the shelf bathymetry. The flow leaving the domain is in geostrophic balance after the transient adjustment of the flow, and so is a steady flow following the straight bathymetry at the open boundary. Since there is no gradient in the bathymetry across the open boundary there is no gradient in the geostrophic velocity across the open boundary and the no-gradient open boundary condition is dynamically consistent with the geostrophic flow. Hence, this boundary condition was used for all runs where the initialization was close to the final adjusted state since the flow at the open boundary was then always close to geostrophic balance.

\subsubsection{Orlanski open boundary condition}

The Orlanski open boundary condition is described in detail by Orlanski (1976) and studied further by Camerlengo and O'Brien (1980) and Chapman (1985). It is designed to let wave-like flows pass though the open boundary with a minimum of distortion and reflected energy. The flow near the open boundary is assumed to be dominated by a single wave described by the simple wave equation

$$
\phi_{t} \pm c \phi_{x}=0
$$

where $\phi$ represents any variable, $t$ is time, $x$ is the cross-boundary coordinate, and $c$ is the phase speed of the wave. Using this wave equation, an estimate of the phase speed of the wave is made just within the open boundary, and then this phase speed is used to project the value of the variable on the open boundary at the next time step. The phase speed is allowed to vary continuously between $\Delta x / \Delta t$ and 0 following 


$$
c=\left\{\begin{array}{cll}
\frac{\Delta x}{\Delta t} & \text { if } & \mp \frac{\phi_{t}}{\phi_{x}} \geq \frac{\Delta x}{\Delta t} \\
\mp \frac{\phi_{t}}{\phi_{x}} & \text { if } & 0<\mp \frac{\phi_{t}}{\phi_{x}}<\frac{\Delta x}{\Delta t} \\
0 & \text { if } & \mp \frac{\phi_{t}}{\phi_{x}} \leq 0 .
\end{array}\right.
$$

There is also a modified version of this boundary condition suggested by Camerlengo and O'Brien (1980) where the phase speed is allowed to be either $\Delta x / \Delta t$ or 0 following

$$
c=\left\{\begin{array}{cl}
\frac{\Delta x}{\Delta t} & \text { if } \mp \frac{\phi_{t}}{\phi_{x}}>0 \\
0 & \text { if } \mp \frac{\phi_{t}}{\phi_{x}} \leq 0
\end{array}\right.
$$

Both versions of this open-boundary condition were used in this study when the initial condition was far from the final adjusted state. The transient response in this case contains eddies and coastally trapped waves which have a large enough amplitude to make the no-gradient open boundary condition inadequate. An Orlanski boundary condition was found to work well in most of these cases. It tended to be less effective when the non-linear terms were large in the momentum equation for high flow speed model runs. The solution to this was to introduce a region near the open boundary where the non-linear terms were made smaller. This allowed the Orlanski boundary condition to work better and did not generate significant upstream disturbance of the flow. When the transient adjustment has decayed away, the Orlanski open-boundary condition is less than ideal since $\phi_{t} / \phi_{x}$ is ill defined. The no-gradient open boundary condition is then preferable. 


\section{Chapter 3}

\section{The Base Case}

\subsection{Introduction}

Various model configurations were used in this study, all of which show a similar adjustment of the flow to the topography. The base case was chosen from amongst these to illustrate the adjustment to the bend in the topography as clearly as possible and to be in a region of parameter space that is more extreme than the conditions over the Scotian Shelf near the Northeast Channel.

A $1 \mathrm{~m} \mathrm{~s}^{-1}$ geostrophically balanced jet, centered on the shelf break, is set to impinge on a sharp, right-hand, bend in the shelf bathymetry ( Figure 3.1). The mean flow over the Scotian Shelf is less than $1 \mathrm{~m} \mathrm{~s}^{-1}$ and the corner in the shelf-break at the Northeast Channel has a radius of $\sim 6 \mathrm{~km}$ (Smith and Petrie, 1982, Schwing, 1992). Hence, the base case is more extreme in both these respects. The jet was chosen so that the inflow decays over the slope and the half width of the jet is narrower than the width of the shelf, so there is no flow near the coast. The flow does not then adjust to the bend in the coastline, and the boundary condition chosen for the coast cannot affect the adjustment of the jet to the bend in the shelf break. The jet is stable to 
small perturbations when flowing parallel to straight bathymetry, it being stabilized by the change in bottom slope at the shelf-break (see Appendix A).

\subsection{The Model Configuration}

The model configuration for the base case is shown in Figure 3.1. The model domain is $200-\mathrm{km}$ wide in the $\mathrm{x}$-direction with 97 grid points and $300-\mathrm{km}$ wide in the $\mathrm{y}$ direction with 145 grid points. This gives a resolution of $2.083 \mathrm{~km}$ in each direction. The time step used was $432 \mathrm{~s}$. The topography has a $100-\mathrm{km}$ wide shelf, with a bottom slope of 0.001 , which goes from $50-m$ deep at the coast to $150-m$ deep at the shelf-break. Beyond the shelf-break, there is then a $60-\mathrm{km}$ wide slope, with a bottom slope of 0.03 , which joins the $150-m$ deep shelf-break to the $1660-m$ deep abyss. The changes of bottom slope from the shelf to the slope and from the slope to the abyss are smoothed using quadratic functions. For a straight section of coastline with the coast at $y=0$, the topography is given by

$$
h(y)= \begin{cases}50+1 y & \text { if } y<100 \mathrm{~km}, \\ 155+1.5(y-100 \mathrm{~km})^{2}-0.05(y-110 \mathrm{~km})^{2} & \text { if } 100<y<110 \mathrm{~km} \\ 305+30(y-110 \mathrm{~km}) & \text { if } 110<y<150 \mathrm{~km} \\ 1660-1.5(y-160 \mathrm{~km})^{2} & \text { if } 150<y<160 \mathrm{~km} \\ 1660 & \text { if } y>160 \mathrm{~km},\end{cases}
$$

where $h$ is the depth in meters and $x$ is the cross-shelf distance in kilometers. One hundred kilometers downstream of the inflow boundary, which runs along $x=0$, the shelf-break turns sharply $90^{\circ}$ to the right and then is straight again to the outflow boundary, which runs along $y=0$.

A Gaussian jet is introduced on the inflow boundary $(x=0)$ between $100<$ $y<300 \mathrm{~km}$, with the maximum velocity centered on the shelf-break, 


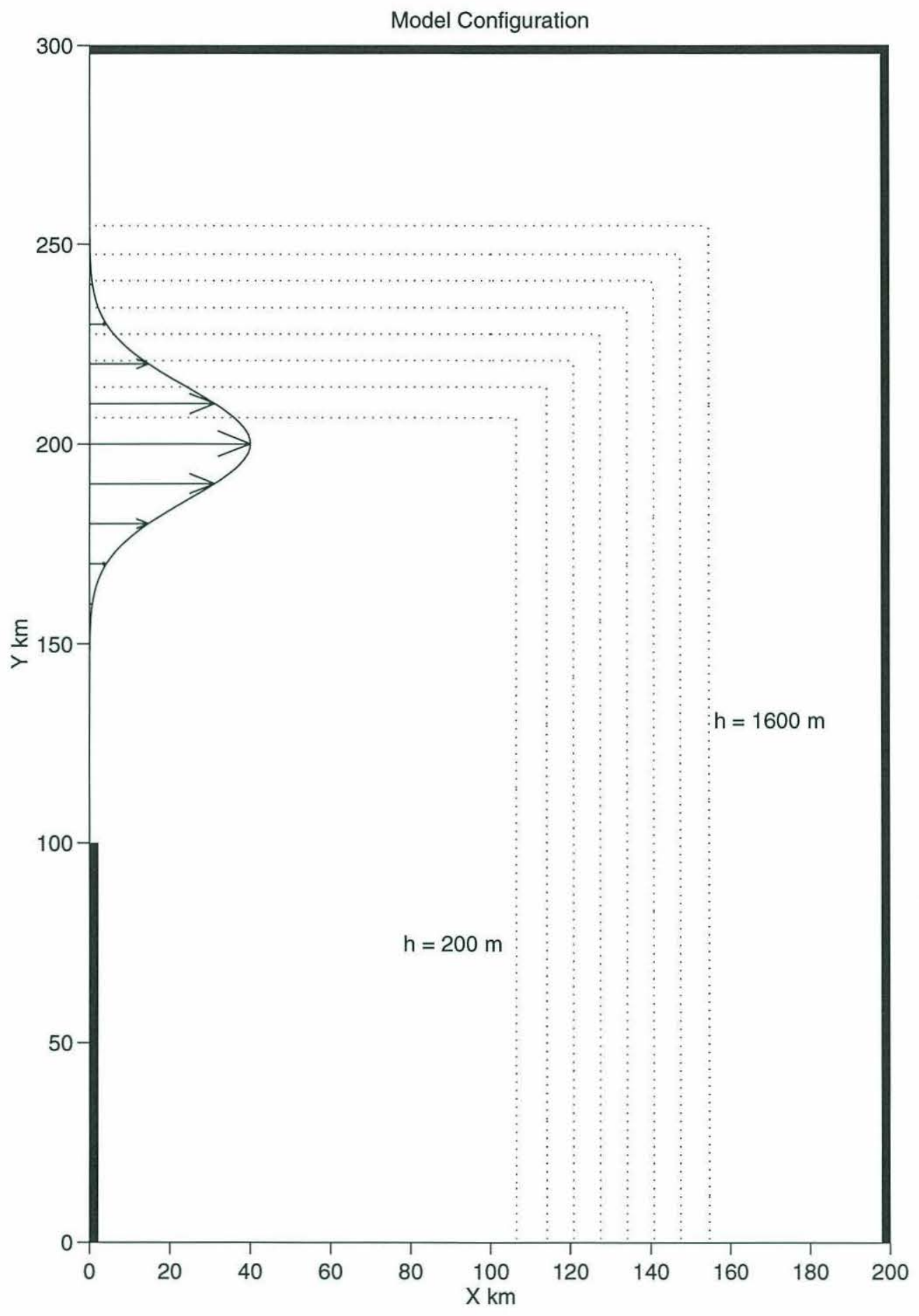

Figure 3.1: An overview of the model configuration for the base case showing the bathymetry (dotted lines) at $200 \mathrm{~m}, 400 \mathrm{~m}, 600 \mathrm{~m}, 800 \mathrm{~m}, 1000 \mathrm{~m}, 1200 \mathrm{~m}, 1400 \mathrm{~m}$, and $1600 \mathrm{~m}$, the free-slip boundaries (thick lines), and the inflow jet. The radius of curvature of the corner is zero and there is no bottom friction. 


$$
u_{\text {inflow }}=u_{\text {max }} e^{-\left(\frac{y-200}{20}\right)^{2}}, \quad v=0 .
$$

The base case has $u_{\max }=1 \mathrm{~ms}^{-1}$ which gives the maximum relative vorticity of the jet to be $4.2 \mathrm{~s}^{-1}$. This jet then flows towards the corner in the shelf topography, and eventually, after adjusting to the presence of the corner leaves the domain through the outflow boundary $y=0$ where the no-gradient open boundary condition is applied. The other boundaries of the domain, $x=0,0 \leq y<100 \mathrm{~km}, x=200 \mathrm{~km}$, and $y=300 \mathrm{~km}$ are free slip boundaries. For example, along $x=200 \mathrm{~km}$, the boundary condition

$$
u=0, \quad v_{y}=0
$$

is applied. The offshore boundaries $(x=200 \mathrm{~km}$ and $y=300 \mathrm{~km})$ are solid for convenience only. The base case was also run with these boundaries as no-gradient, open boundaries and whilst this proved to be unstable over the abyss, with flow into the domain along $y=300 \mathrm{~km}$ and flow out of the domain along $x=200 \mathrm{~km}$, the flow over the shelf remained essentially unchanged and so the presence of closed offshore boundaries does not affect the adjustment of the inflow jet to the corner in the shelf break.

The base case is a frictionless run so the linear bottom friction coefficient $r_{f}$ is set to zero.

\subsection{Description of the Flow}

This model run has no bottom friction and the horizontal viscosity, introduced to damp numerical noise, is dynamically insignificant. The forcing of the flow is then solely through the depth-independent forcing from the surface pressure gradient on the rigid lid which produces a depth-independent acceleration since the density of the flow is constant. The inflow velocity, which is initially depth-independent, will remain 
depth independent with depth-independent acceleration and this is found to be the case on examining the flow field. The governing equations for the flow are then the shallow water equations

$$
\begin{aligned}
& \frac{\partial u}{\partial t}+u \frac{\partial u}{\partial x}+v \frac{\partial u}{\partial y}-f v=-\frac{1}{\rho_{0}} \frac{\partial P}{\partial x} \\
& \frac{\partial v}{\partial t}+u \frac{\partial v}{\partial x}+v \frac{\partial v}{\partial y}+f u=-\frac{1}{\rho_{0}} \frac{\partial P}{\partial y}
\end{aligned}
$$

and the appropriate form of the potential vorticity $\Pi$ is

$$
\Pi=\frac{f+\omega}{h},
$$

where $\omega$ is the relative vorticity defined by $v_{x}-u_{y}$.

Figures $3.2,3.3,3.4$, and 3.5 show various properties of the flow field after the transient adjustment to the initial conditions has decayed and will be discussed in detail in the following paragraphs. The flow is steady and the streamlines are very nearly symmetrical about the corner. The inflow jet does not flow to the corner in the shelf-break and overshoot, even with the radius of curvature of the corner equal to zero, but adjusts to the presence of the corner before the corner is reached and turns the corner smoothly. This illustrates that the inertial radius criterion given by Klinger (1994) for the separation of flow from a coast does not apply here.

The flow shoals as it approaches the corner and then deepens again as it flows away from the corner. The shoaling and deepening of three streamlines are shown in Figure 3.6. The depth of a streamline is very nearly symmetrical about the corner, and so far downstream of the corner the flow returns to close to its initial upstream depth. The relative change of depth of a streamline is defined by $\left(h_{0}-h\right) / h_{0}$ where $h_{0}$ is the initial depth and $h$ is the current depth. Over the shelf, the relative change of depth at the corner is $\sim 0.1$, whereas over the slope the depth changes much more sharply, the relative change of depth reaching a maximum of 0.52 near the top of the slope. 
(a)

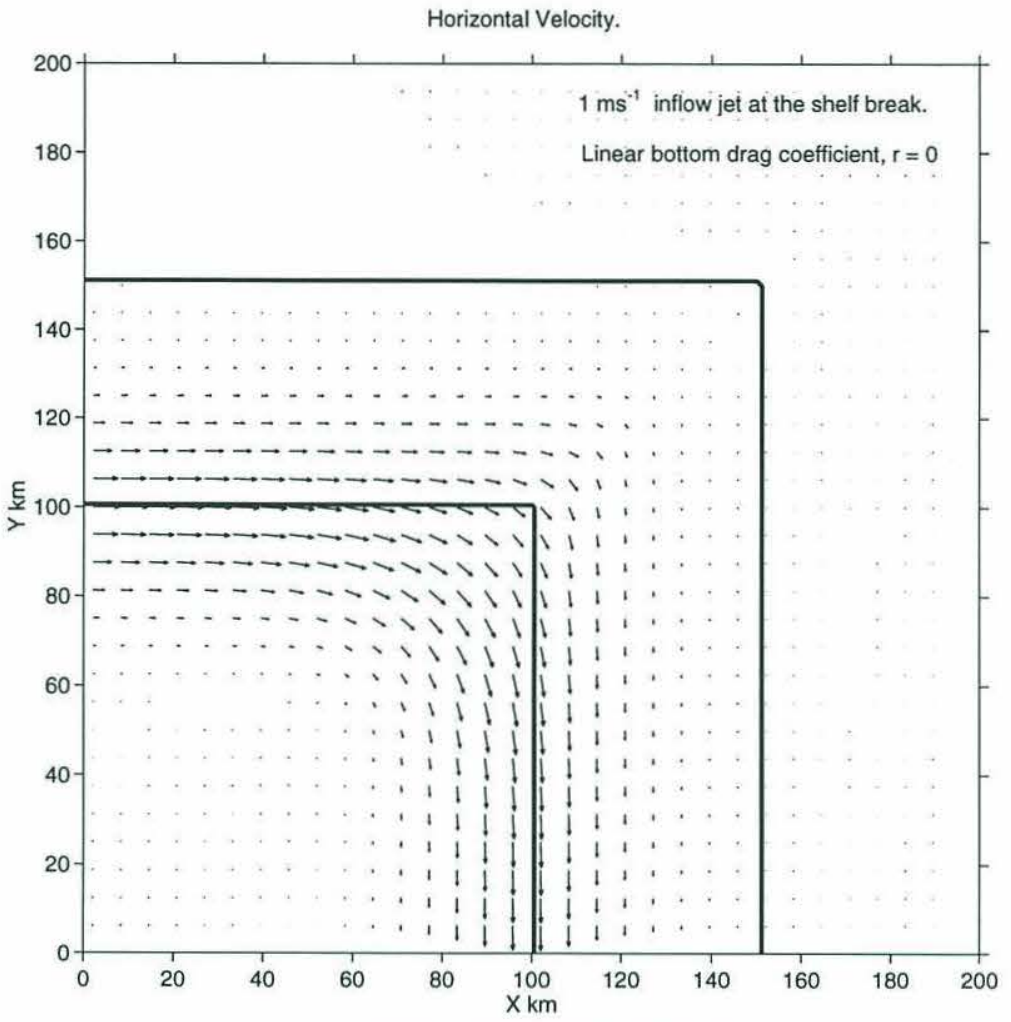

(b)

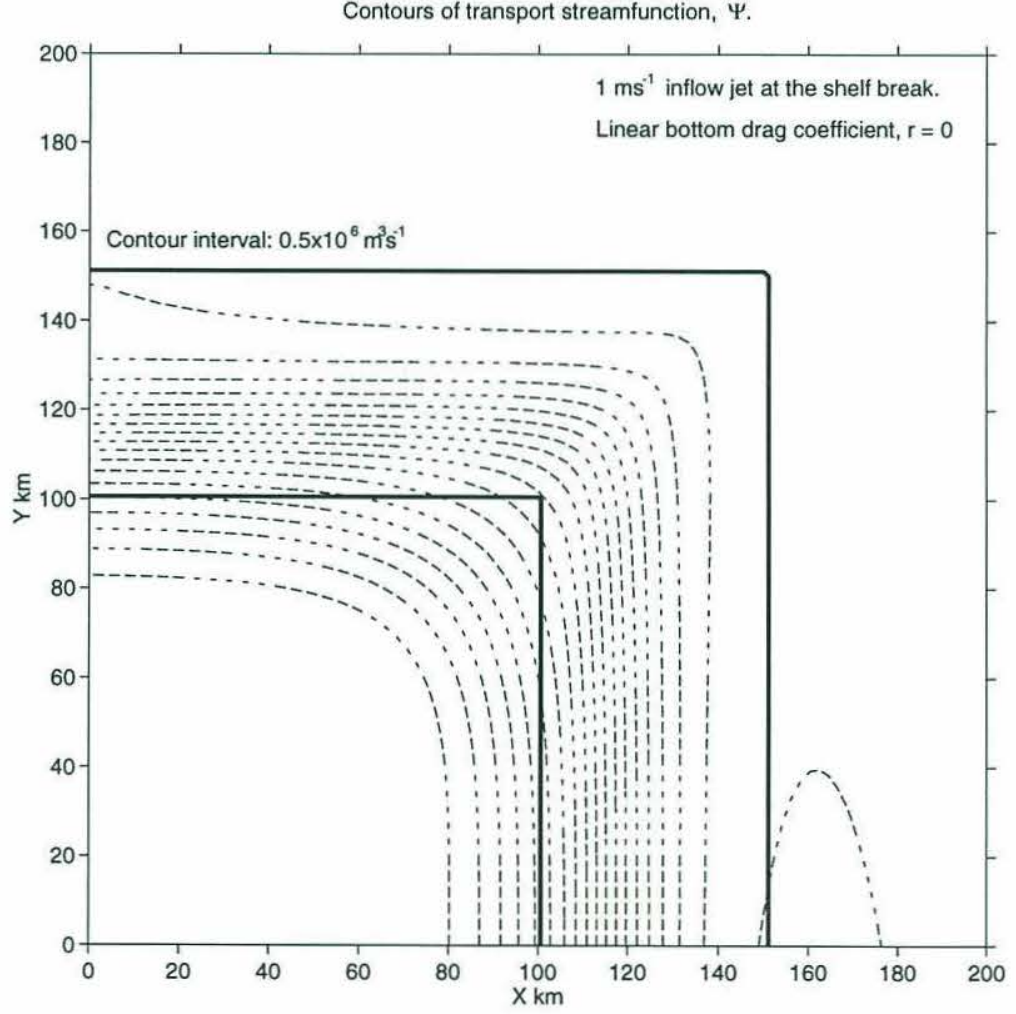

Figure 3.2: The base case: (a) horizontal velocity, (b) transport streamfunction. 
(a)

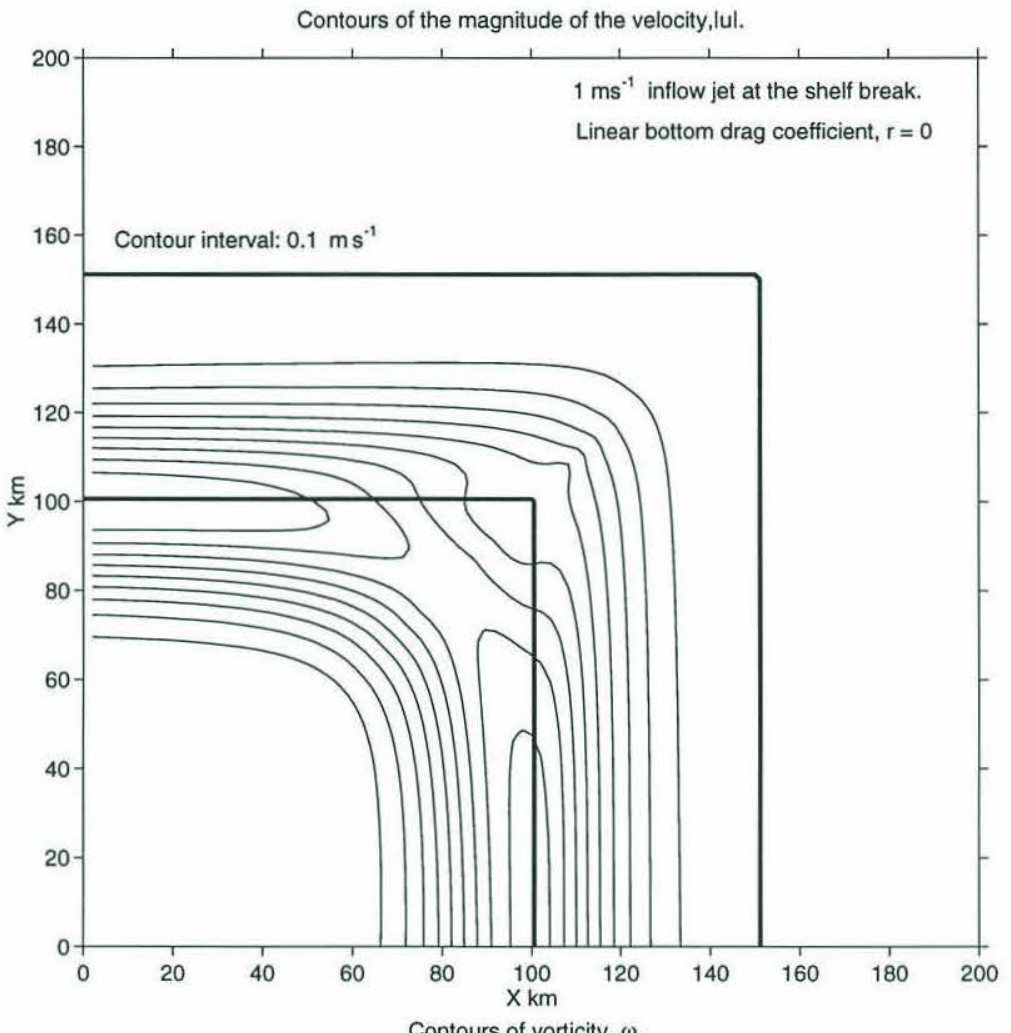

(b)

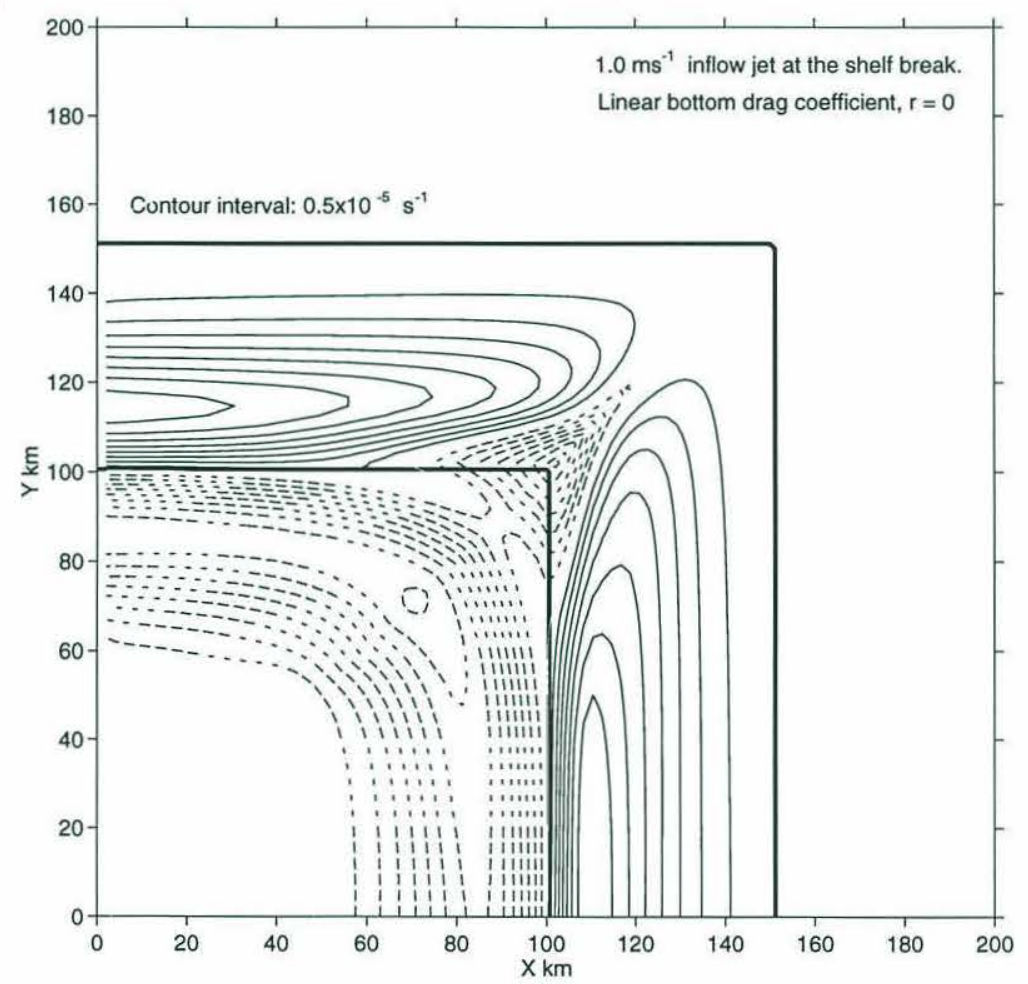

Figure 3.3: The base case continued: (a) magnitude of the velocity, (b) vorticity. 
(a)

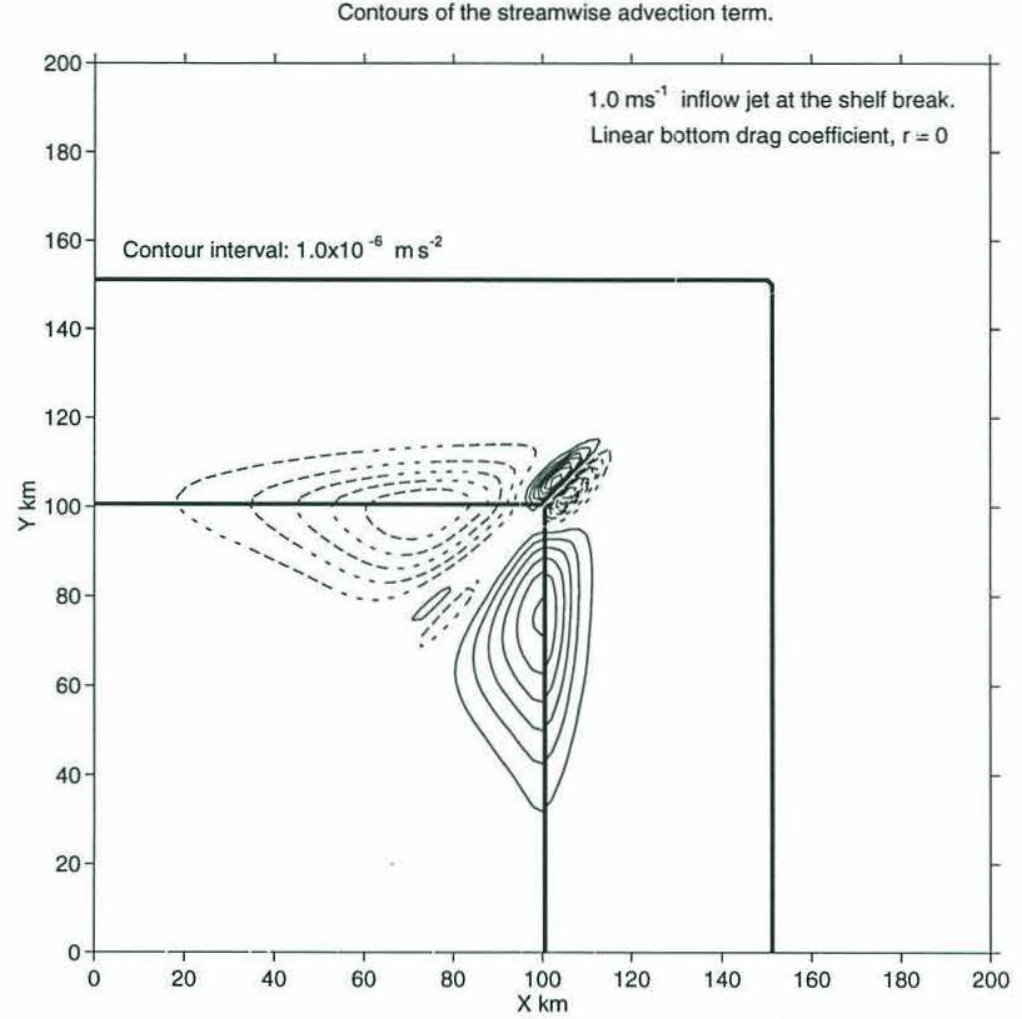

(b)

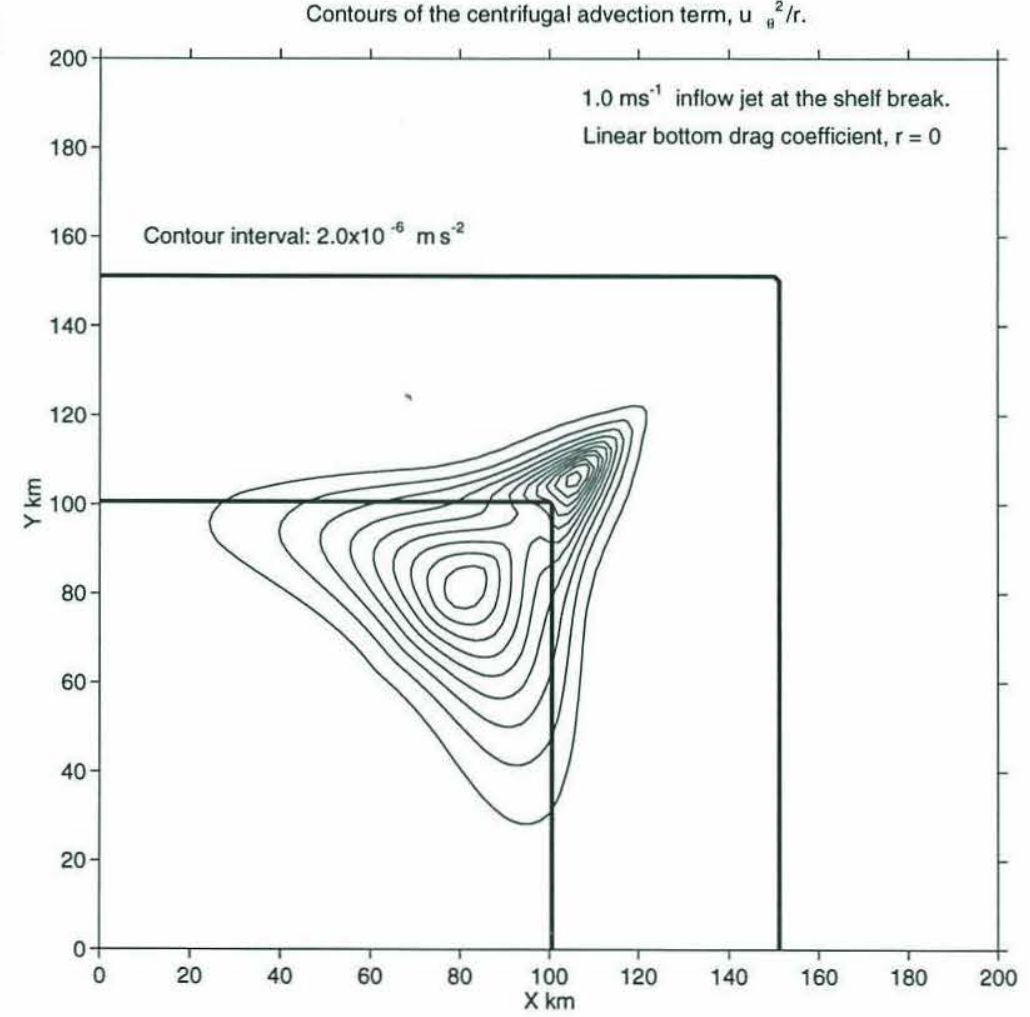

Figure 3.4: The base case continued: (a) streamwise advection, (b) centrifugal advection. 
(a)

Contours of the radius of curvature of the flow, $r$, near the corner.

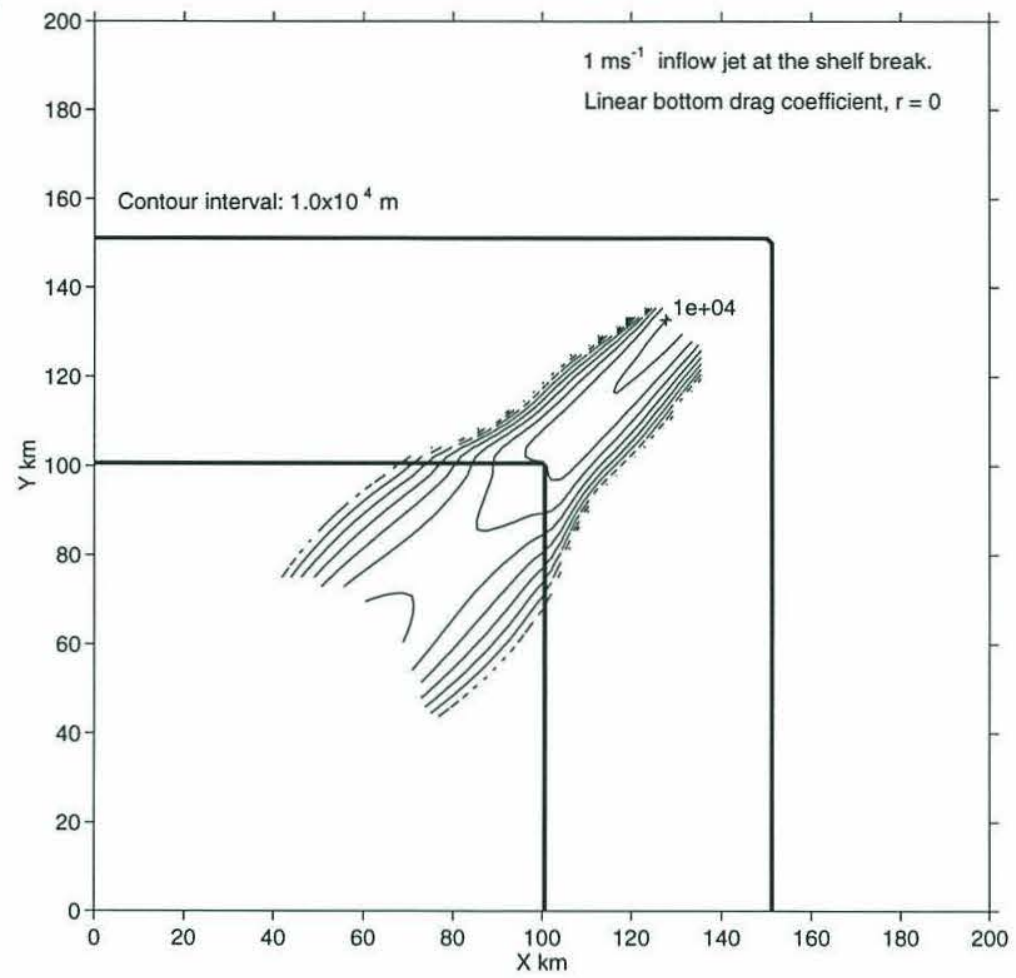

(b)

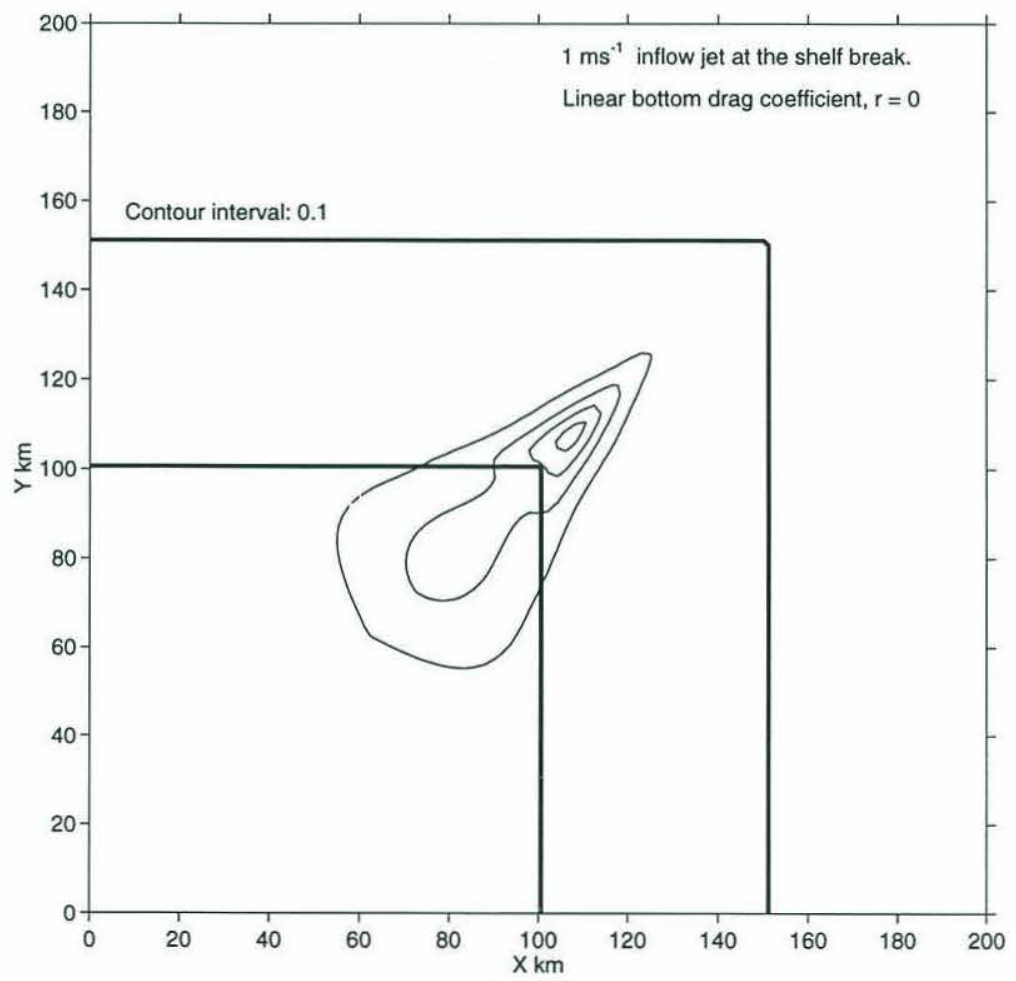

Figure 3.5: The base case continued: (a) radius of curvature, (b) local Rossby number. 


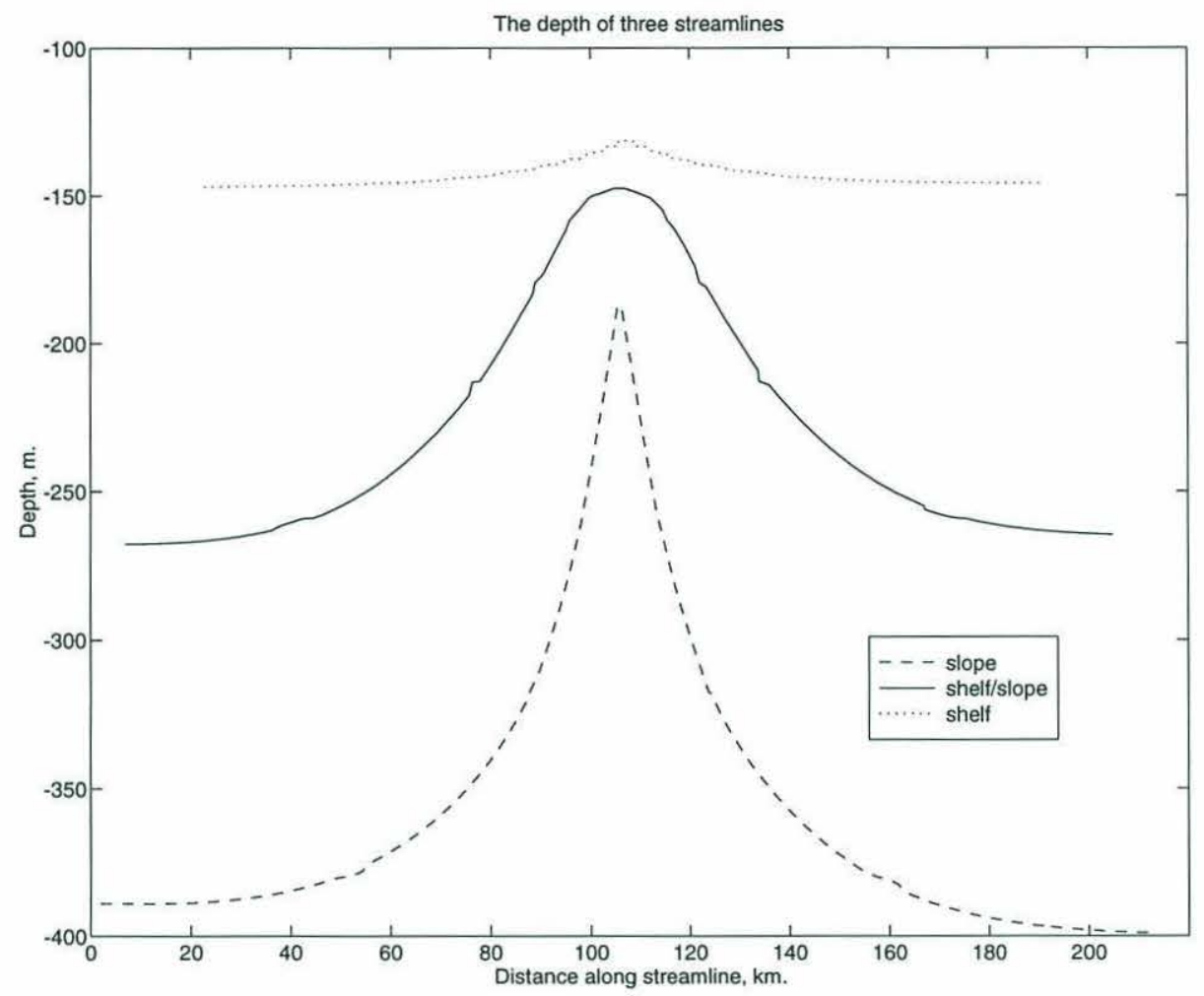

Figure 3.6: The depth along three streamlines plotted as a function of distance along the streamline. One streamline is located entirely over the slope (dashed line), one entirely over the shelf (dotted line), and one over the slope far from the corner and over the shelf near the corner (solid line).

The flow that is over the shelf at the corner is weakly constrained horizontally in comparison with that which is over the slope. This is because the bottom slope over the shelf is $1 / 30$ of the bottom slope over the slope, so, for a given amount of vortex stretching, the horizontal displacement is 30 times greater. Hence, the flow adjusts in a much broader fashion over the shelf, the radius of curvature at the corner being $\sim 35 \mathrm{~km}$ over the shelf compared with $\sim 15 \mathrm{~km}$ over the slope (see Figure 3.5 (a)).

Near the corner, the flow deviates from the linear solution which follows lines of $f / h$, and the non-linear terms in the momentum equation are found to be significant. To consider the acceleration of a fluid column as it turns the corner, it is useful to use streamwise coordinates to resolve the acceleration into an along-stream (or 
streamwise) component and a cross-stream (or centrifugal) component. In streamwise coordinates, the shallow water equations (3.4) and (3.5) become, for a steady flow

$$
\begin{aligned}
u_{s} \frac{\partial u_{s}}{\partial s} & =-\frac{1}{\rho_{0}} \frac{\partial P}{\partial s} \\
-\frac{u_{s}^{2}}{r}+f u_{s} & =-\frac{1}{\rho_{0}} \frac{\partial P}{\partial n}
\end{aligned}
$$

where $s$ is the along-stream coordinate, $n$ is the cross-stream coordinate, and $u_{s}$ is the along-stream velocity. Figures 3.4 (a) and (b) show the streamwise advection term and the centrifugal advection term. The flow broadens to 1.5 times its inflow width as it approaches the corner so that, although it is shoaling, there is a wide region of streamwise deceleration that is centered on the shelf-break before the corner. This deceleration, reaching a minimum of $\sim-6 \times 10^{-6} \mathrm{~m} \mathrm{~s}^{-2}$, reduces the maximum speed of the jet to $0.79 \mathrm{~m} \mathrm{~s}^{-1}$ at the corner (see Figure 3.7 (a)). In keeping with the symmetry of the flow, there is a similar region of streamwise acceleration as the flow leaves the corner. Thus the shoaling of the jet almost compensates for the broadening of the jet, and the streamwise velocity is roughly constant following a streamline.

The centrifugal advection term $u_{s}^{2} / r$ is the acceleration perpendicular to the flow due to the curvature of the flow, and so reaches a maximum at the corner. The maximum centrifugal advection is $22 \times 10^{-6} \mathrm{~m} \mathrm{~s}^{-2}$ which occurs at the corner over the slope where the flow is turning with a small radius of curvature of $15 \mathrm{~km}$ and moving at $0.5 \mathrm{~m} \mathrm{~s}^{-1}$. There is a smaller maximum in the centrifugal advection of $18 \times 10^{-6} \mathrm{~m} \mathrm{~s}^{-2}$ which occurs at the corner over the shelf where the flow is turning with a larger radius of curvature of $35 \mathrm{~km}$ and moving at $0.79 \mathrm{~m} \mathrm{~s}^{-1}$.

The local Rossby number, calculated as $u_{s} / f r$, reaches 0.4 at the corner over the slope. It is the broad adjustment and shoaling of the flow that prevents $u_{s} / f r$ reaching 1. This is fundamentally different from the results of Klinger (1994), where at the corner in the coast, the local Rossby number is greater than one. It is the broad shelf, which allows the broad adjustment near the shelf break, that leads to 
the small local Rossby number in the case considered here. This kind of adjustment is not possible for the experiments in Klinger (1994) because of the presence of the coast.

\subsection{Vorticity Dynamics}

The dynamical balance of this flow is best understood through the conservation of shallow water potential vorticity. Since the flow is steady, and the frictional terms are zero or negligible, the conservation of potential vorticity is expressed as

$$
u \frac{\partial \Pi}{\partial x}+v \frac{\partial \Pi}{\partial y}=0 .
$$

Potential vorticity is then constant along streamlines and the model results were checked for this.

The vorticity field for this flow is shown in Figure 3.3 (b). The fluid first gains and then loses negative relative vorticity as it shoals and deepens in accordance with (3.9). The maximum change in relative vorticity coincides with the greatest change in relative depth, which occurs over the top of the slope. Figure 3.7 (b) shows the relative vorticity of the inflow jet and the relative vorticity of the jet at the corner. The large gain in negative relative vorticity over the top of the slope can clearly be seen. In streamwise coordinates, the relative vorticity is written

$$
\omega=-\frac{u_{s}}{r}-\frac{\partial u_{s}}{\partial r} .
$$

The first term on the right-hand side of (3.10) is the relative vorticity due to the turning of the flow and the second term is the relative vorticity due to the shear in the flow. The dotted line in Figure 3.7 (b) is the relative vorticity due to the shear of the flow at the corner. The difference between this and the total relative vorticity is the relative vorticity due to the curvature of the flow. It can be seen that on the right-hand side of the jet, which is over the shelf, the total relative vorticity changes 


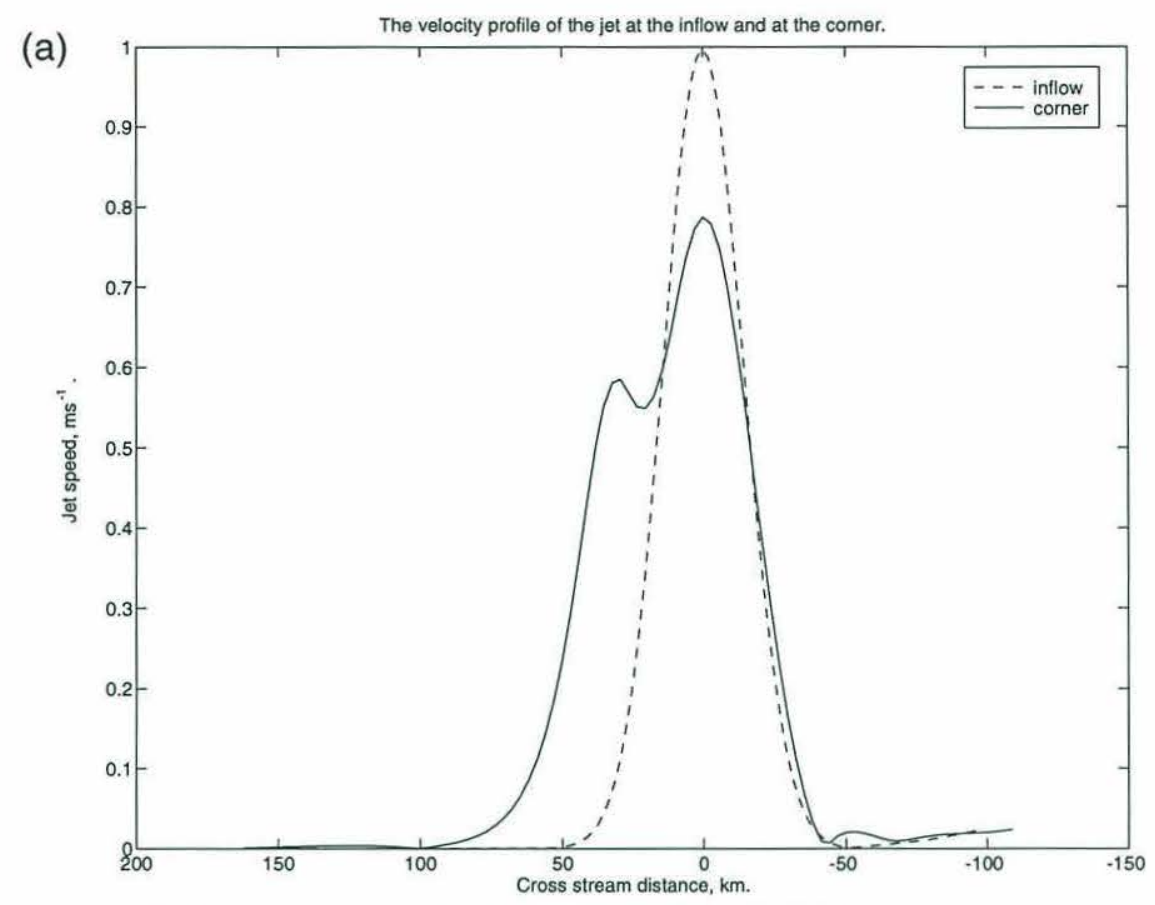

(b)

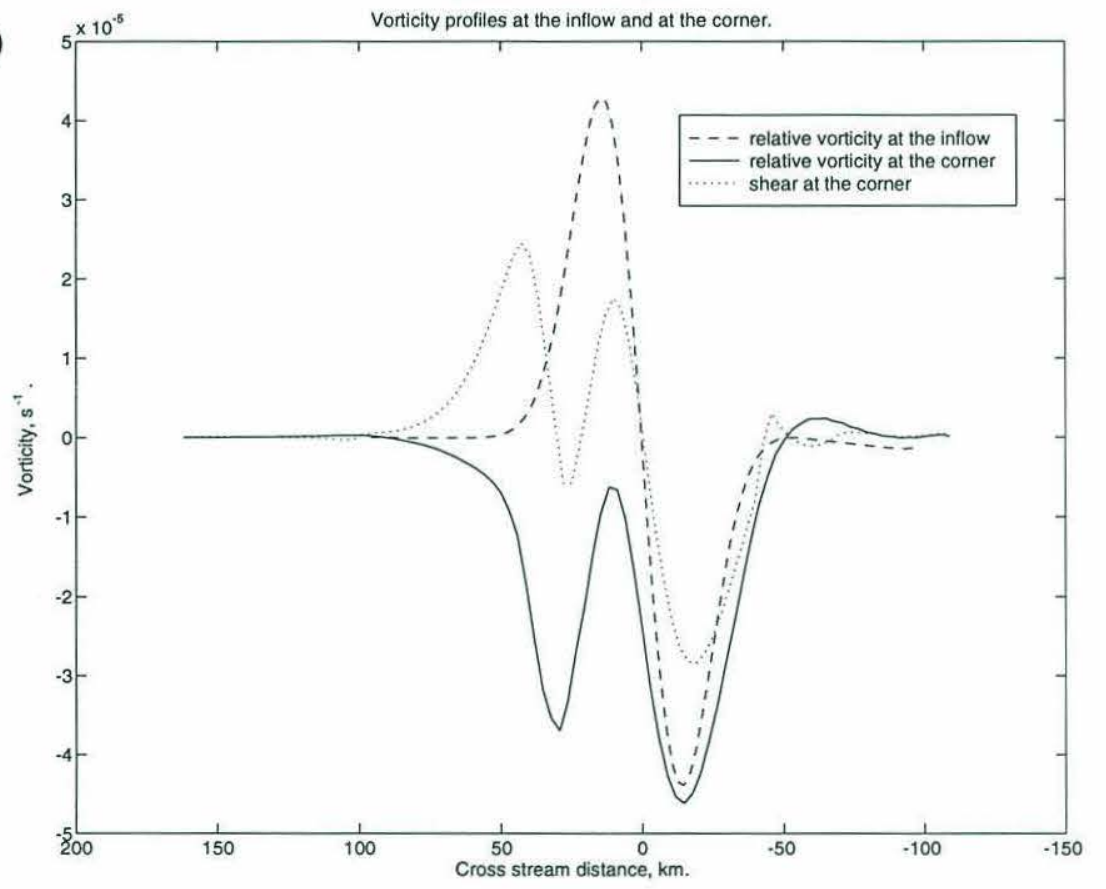

Figure 3.7: (a) The speed of the jet against distance across the jet at the inflow (dashed line), and at the corner (solid line). (b) The relative vorticity of the jet against distance across the jet at the inflow (dashed line), and at the corner (solid line). The dotted line is the relative vorticity due to the shear in the flow at the corner. Both Figures are looking downstream with the shelf on the right and the slope on the left. 
little and the decrease in the size of the shear of the flow, due to its deceleration, provides the increase in curvature. On the left side of the jet a reduction in the size of the shear and an increase in the curvature both cause a gain in negative relative vorticity so the the adjustment is more dramatic with a larger change in both the shear and the curvature of the flow.

The dynamical balance is consistent with the following vorticity argument. If a fluid column is followed from the inflow jet, as the flow begins to turn the corner it becomes shallower, and so, through conservation of potential vorticity, must gain negative relative vorticity ( see Figure 3.3 (b)). If we consider that this negative vorticity is manifested, crudely, in the turning of the jet rather than the shear of the jet, then the flow will continue to turn as it approaches the corner and so continue to shoal. On passing the corner, the argument is reversed. The flow is now heading into deeper water so the fluid column now loses its negative relative vorticity and turns less. Far from the corner the flow stops turning and follows lines of $f / h$, once again.

A similar balance is not possible if the flow is reversed, i.e., when the coast is on the left of the inflow jet rather than the right. In this case, if the flow shoals before the corner, the gain in negative relative vorticity will tend to put the jet in deeper water again if it is manifested in the turning of the jet. The tendency will be for the jet to flow towards the corner rather than to turn before it.

For the base case, with the coast on the right of the jet, the direction of propagation of long-wavelength coastally trapped vorticity waves is with the flow and so perturbations in the vorticity field near the corner will always tend to propagate downstream. However, if the coast is on the left of the jet, perturbations in the vorticity field at the corner will tend to propagate upstream for low flow speeds and then, if the flow speed is increased, the perturbations would become trapped at the corner. It is expected that at this point the dynamics of the flow around the corner would change and the flow would separate from the bathymetry. This illustrates an 
additional difference between having the coast on the left and having the coast on the right.

\subsection{A Simple Model}

To gain further insight into the vorticity dynamics, a simple analytical model is presented here which builds on the vorticity arguments of the previous section.

A schematic of the model is shown in Figure 3.8 (a). A thin, barotropic jet with a 'top-hat' profile is set to run parallel to bathymetry which slopes upwards to the right with a constant bottom-slope $b$, and surfaces a distance $L$ from the jet. The initial depth of the jet is $h_{0}$, so $h_{0}=b L$. As in the base case, there is a sharp bend to the right in the bathymetry far downstream of the initial conditions and the jet must adjust to it as it approaches.

Assuming the jet shoals as it approaches the corner, it must gain negative relative vorticity. The assumption is made that the shear in the flow is initially zero and remains zero at all times, so the relative vorticity is now

$$
\omega=\frac{-u_{s}}{r}
$$

and all the negative relative vorticity is in the curvature of the jet. The equation for potential vorticity conservation can then be written

$$
\frac{u_{s}}{r}=\frac{f\left(h_{0}-h\right)}{h_{0}} .
$$

A particularly simple continuity equation is chosen

$$
u_{s}=\text { constant }
$$

which dictates that as the jet shoals, it must broaden to maintain a constant speed. The base case flow roughly does this. 
(a)

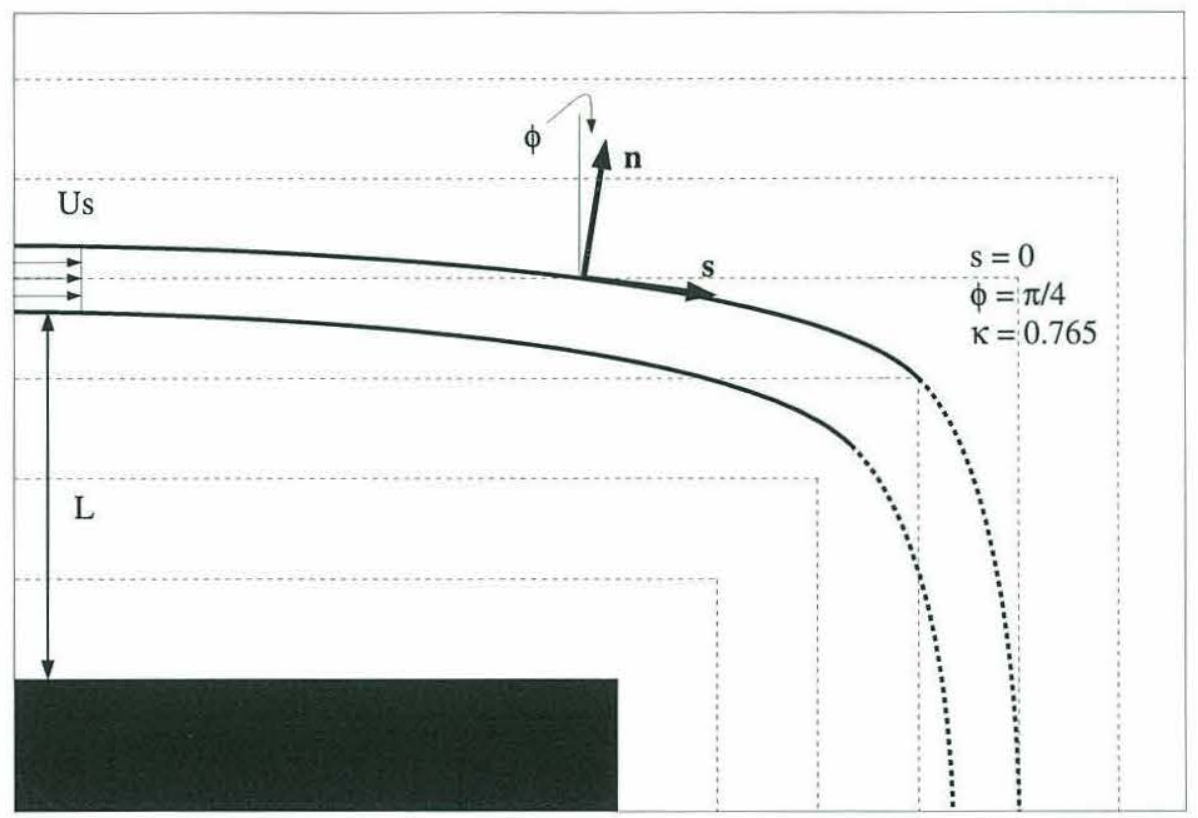

(b)

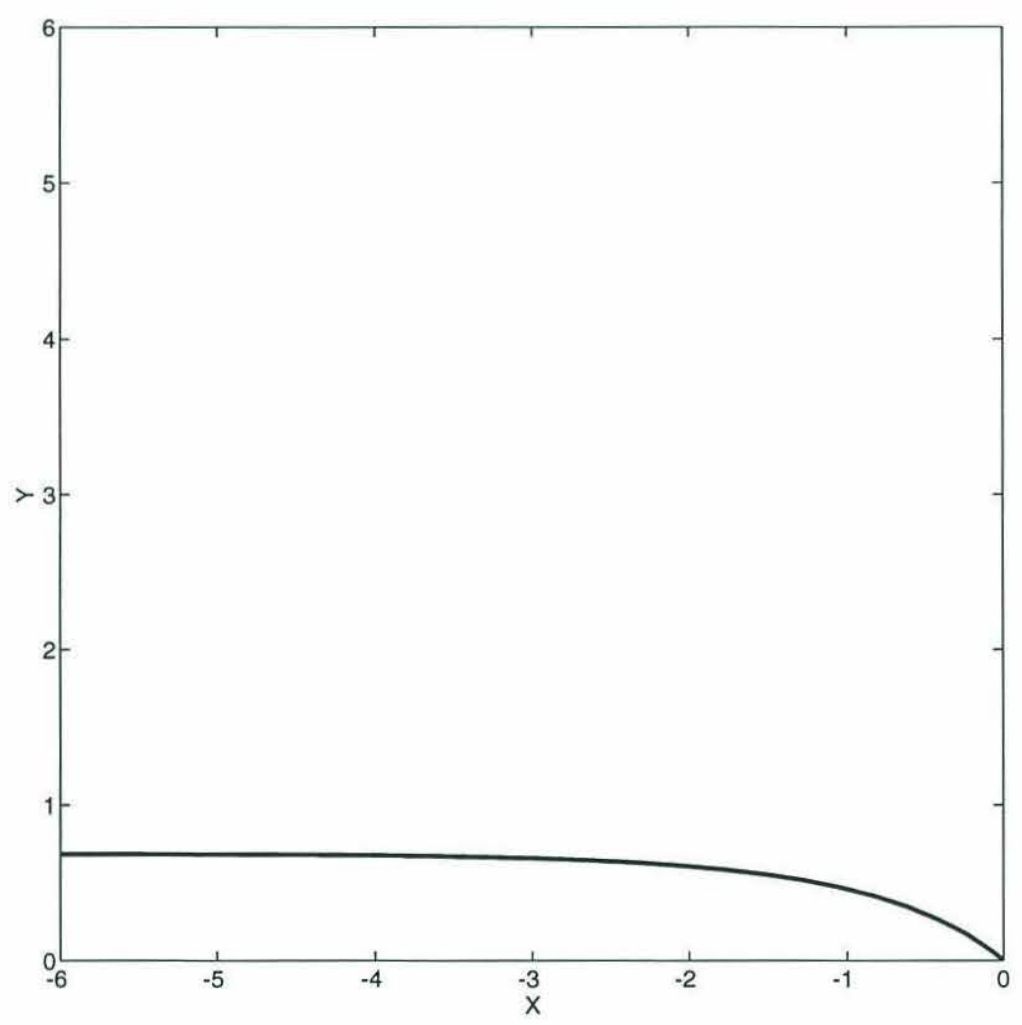

Figure 3.8: (a) A schematic of the simple model. (b) The path of the jet for the non-dimensional, scaled solution to the simple model. The X-axis runs along-shore and the $\mathrm{Y}$-axis runs off-shore. The axes are marked in the non-dimensional, scaled length $l$. 
The rate of change of depth with distance along the jet is given by

$$
\frac{d h}{d s}=-b \sin \phi,
$$

where $\phi$ is the angle the jet has turned through.

The substitution $1 / r=d \phi / d s$ is made in (3.12) and then equations (3.12) and (3.14) are non-dimensionalized, writing $s^{*}=L s$ and $h^{*}=h_{0} h$, to get

$$
\begin{aligned}
\epsilon \frac{d \phi}{d s} & =1-h \\
\frac{d h}{d s} & =-\sin \phi
\end{aligned}
$$

where the starred variables are now the dimensional ones, and $\epsilon=u_{s} / f L$ is the Rossby number for the flow.

Differentiating (3.15) with respect to $s$ and substituting for $d h / d s$ using (3.16) yields an equation for the path of the flow

$$
\frac{d^{2} \phi}{d s^{2}}=\frac{1}{\epsilon} \sin \phi,
$$

which is similar to the path equation given by Robinson and Niiler (1967).

$\phi$ and $\sin \phi$ are both $O(1)$ numbers for this problem so it is appropriate to scale $s$ with the square-root of the Rossby number, $s=\sqrt{\epsilon} l$, which gives the equation

$$
\frac{d^{2} \phi}{d l^{2}}=\sin \phi
$$

Because of the symmetry of the flow, this equation can be solved by shooting back towards the inflow from the corner with the initial conditions

$$
\phi=\frac{\pi}{4}, \quad \frac{d \phi}{d l}=\kappa_{0} \quad \text { at } \quad l=0 .
$$

When $\kappa_{0}$ is chosen correctly, $\phi \rightarrow 0$ as $s \rightarrow-\infty$. This value of $\kappa_{0}$ is $\sim 0.765$ and the solution obtained is shown in Figure 3.8 (b). 


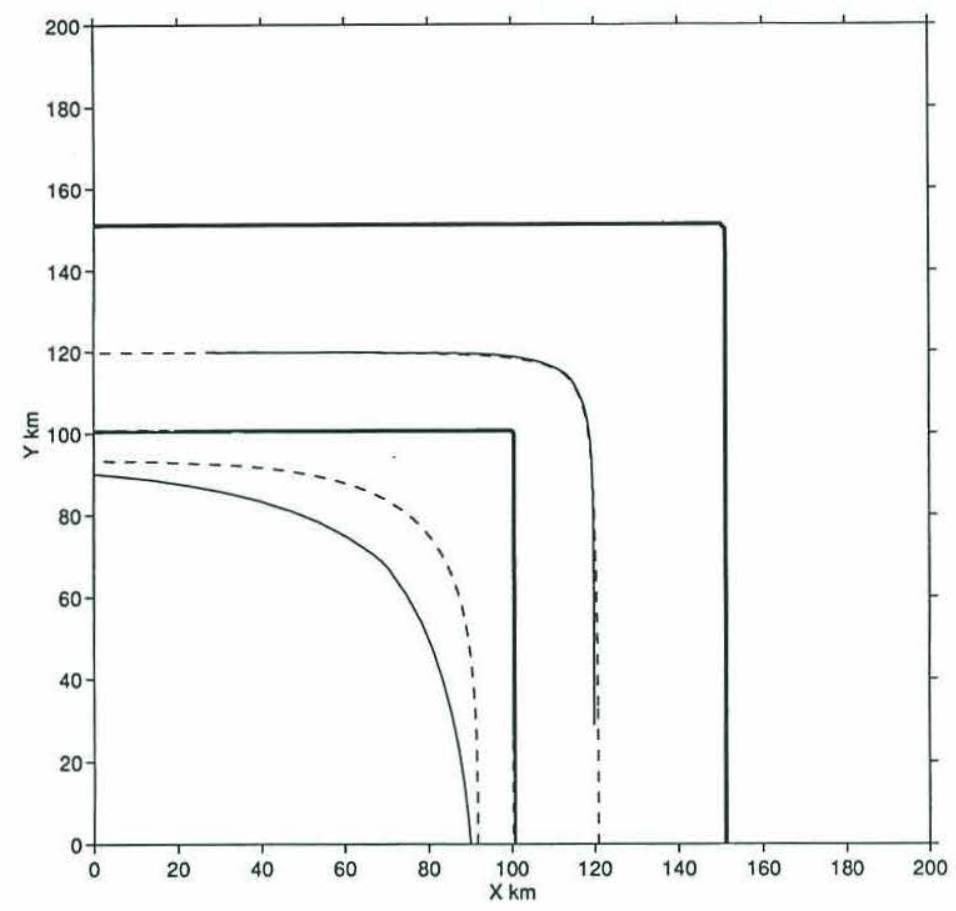

Figure 3.9: Two streamlines from the base case (dotted lines) and the corresponding streamlines from the simple model (solid lines).

The solution was rescaled to fit the base case model run for various positions across the inflow jet. $\epsilon$ varies across the inflow jet as the speed of the jet varies and the distance to where the local bottom slope would surface varies. Note that, because of the $\sqrt{\epsilon}$ scaling, the flow turns more sharply for low $\epsilon$ and more broadly for large $\epsilon$. Figure 3.9 shows a comparison between two streamlines from the base case, one over the shelf and one over the slope, and the simple model. The base case flow turns more sharply over the shelf than the simple model solution does because some of the relative vorticity due to the turning of the flow is from the reduction of the size of the shear on the right-hand side of the jet. Over the slope the base case and the simple model compare very well.

The non-dimensionalisation and scaling that lead to this equation give the appropriate scaling for the flow at the corner and this will be discussed in the next chapter. 


\section{Chapter 4}

\section{Scalings and Parameter Variations}

\subsection{Introduction}

The parameters for the base case were varied to explore the nature of the adjustment at the corner for oceanographically reasonable parameters. The simplest way to alter the Rossby number of the flow for the base case is to vary the inflow velocity. A series of runs show the adjustment at the corner for a range of inflow speeds from $0.1 \mathrm{~m} \mathrm{~s}^{-1}$ to $2.0 \mathrm{~m} \mathrm{~s}^{-1}$. The simple model of the previous chapter is then used to give a scaling for the flow at the corner.

The model configuration for the base case was chosen so that the resulting dynamics were as simple as possible. The sharp corner, inviscid dynamics and jet inflow are to some extent unrealistic of the Scotian Shelf, so dependence of the flow on these aspects of the base case was examined in order to check the robustness of the results. 


\subsection{Inflow Velocity}

Figures 4.1 and 4.2 shows the adjustment at the corner for a series of runs with inflow jet speeds of $0.1 \mathrm{~m} \mathrm{~s}^{-1}, 0.5 \mathrm{~m} \mathrm{~s}^{-1}, 1.0 \mathrm{~m} \mathrm{~s}^{-1}$, and $2.0 \mathrm{~m} \mathrm{~s}^{-1}$. The runs were otherwise identical to the base case. The flow is steady and adjusts to the corner in a similar manner to the base case. The $0.1 \mathrm{~m} \mathrm{~s}^{-1}$ jet has essentially linear dynamics, and turns the corner very sharply with the local Rossby number reaching a maximum of 0.1 at the corner. As the flow speed is increased, the symmetry of the flow is maintained and the flow adjusts in a broader manner, the radius of curvature of the flow increasing with increasing flow speed. For the $2.0-\mathrm{m} \mathrm{s}^{-1}$ jet, the maximum radius of curvature at the corner is $50 \mathrm{~km}$ and the local Rossby number reaches 0.62 , which is less than one due to the broad nature of the adjustment.

\subsection{Scaling}

For an inviscid Gaussian jet flowing parallel to straight bathymetry of constant bottom slope $b$, there are two length scales, the distance $L$ from the center of the jet to the coast where the bathymetry surfaces, and the width of the jet $W$. The velocity scale is taken to be the maximum speed of the jet $u_{\max }$ and the rotational time scale is $1 / f$.

The two relevant non-dimensional parameters for this problem are the Rossby number $\epsilon$ and the ratio of the two length scales $\alpha$

$$
\epsilon=\frac{u_{\max }}{f L}, \quad \alpha=\frac{W}{L}
$$

Note that it is not necessary to consider the bottom slope since it is the relative depth of a fluid column that is important to the vorticity dynamics and this does not depend on the bottom slope. 
(a)

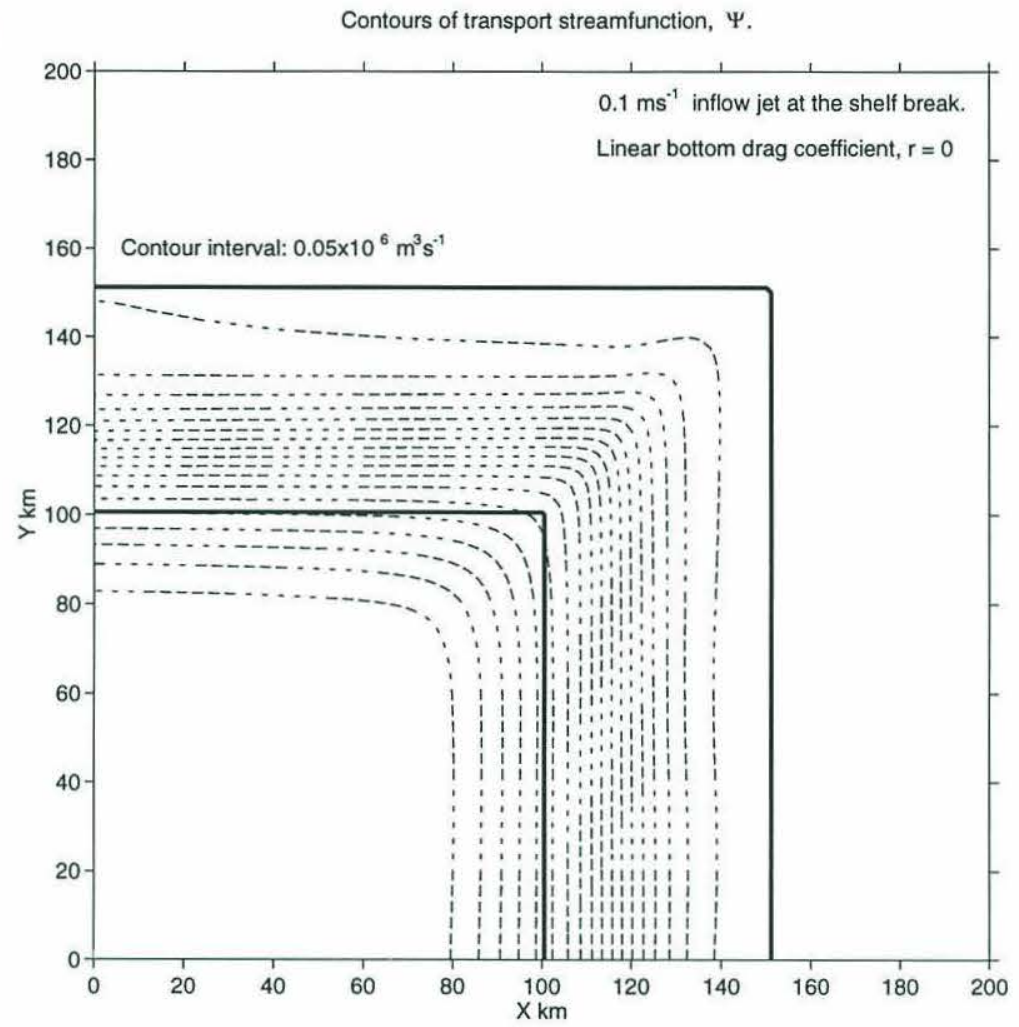

(b)

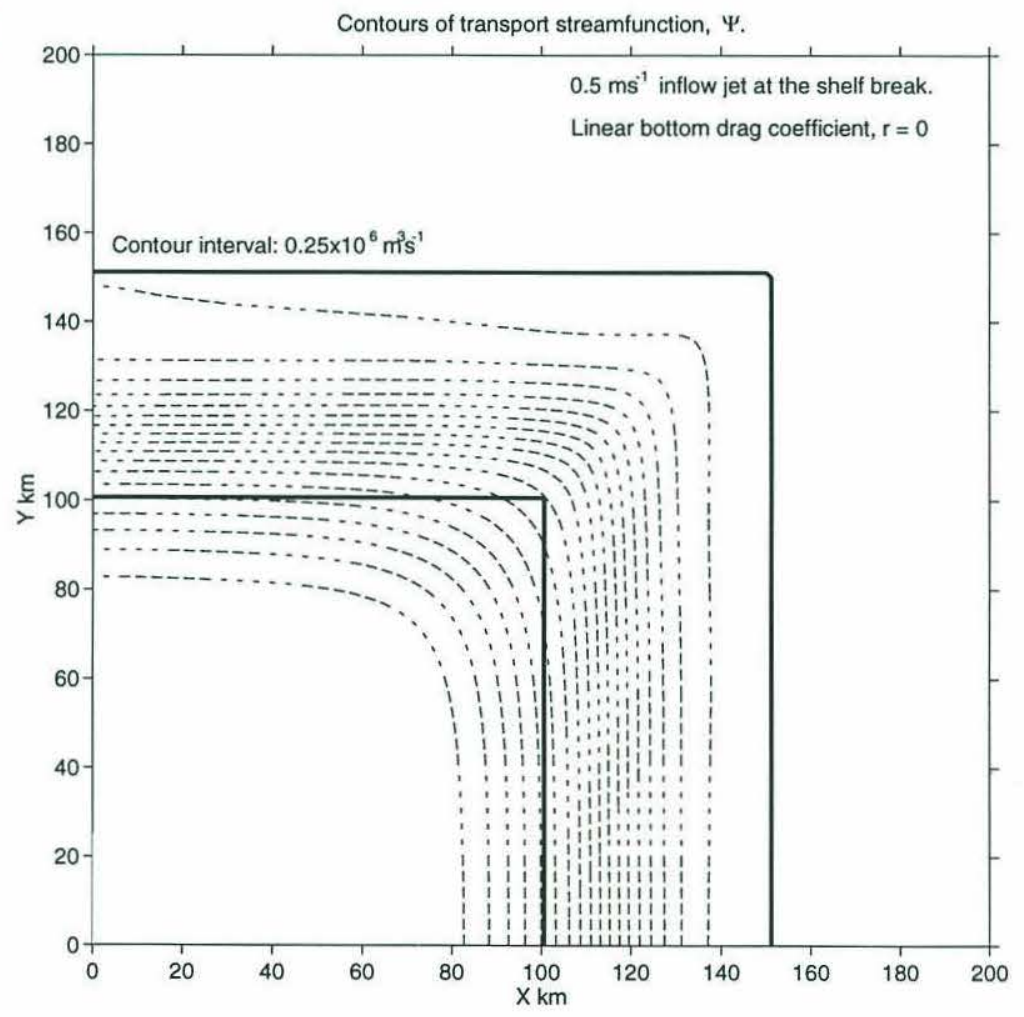

Figure 4.1: Contours of the transport streamfunction for the base case with varying inflow speeds (a) $0.1 \mathrm{~m} \mathrm{~s}^{-1}$ (b) $0.5 \mathrm{~m} \mathrm{~s}^{-1}$. 
(a)

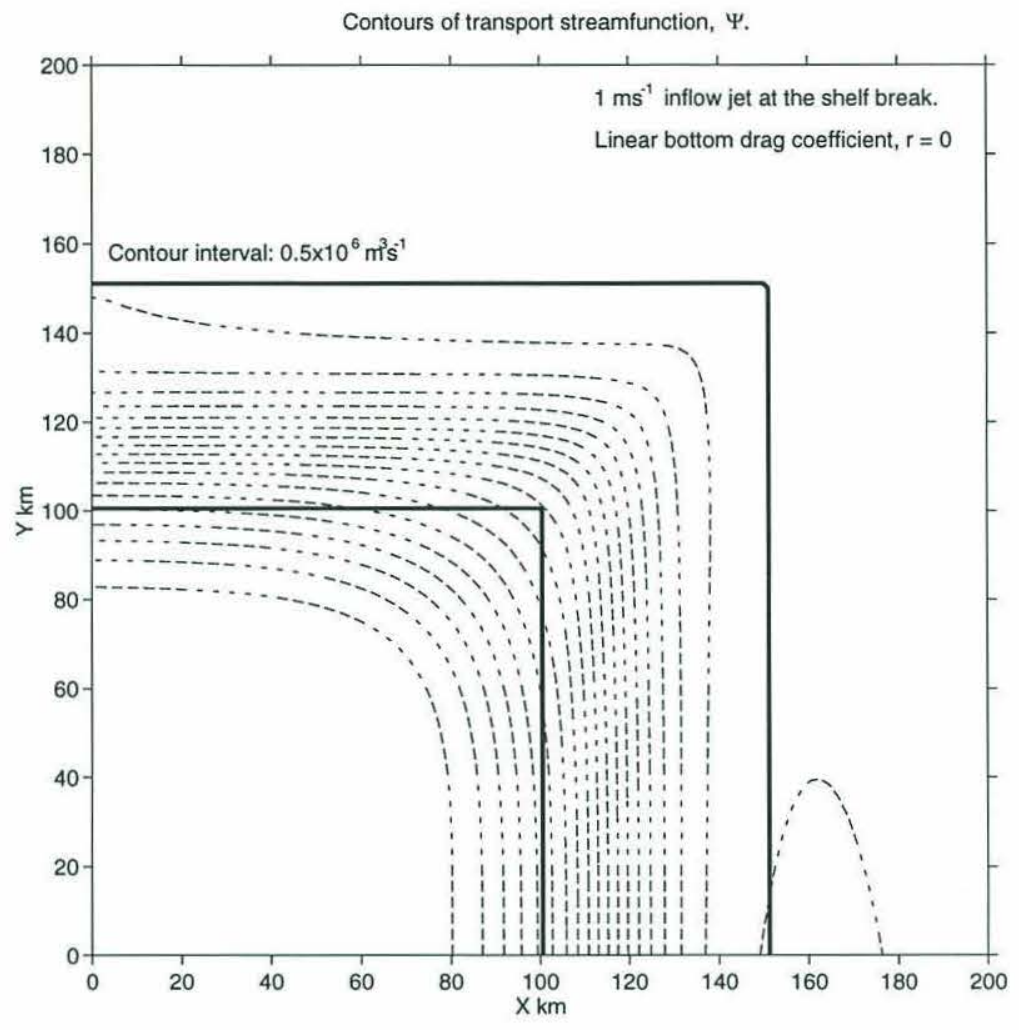

(b)

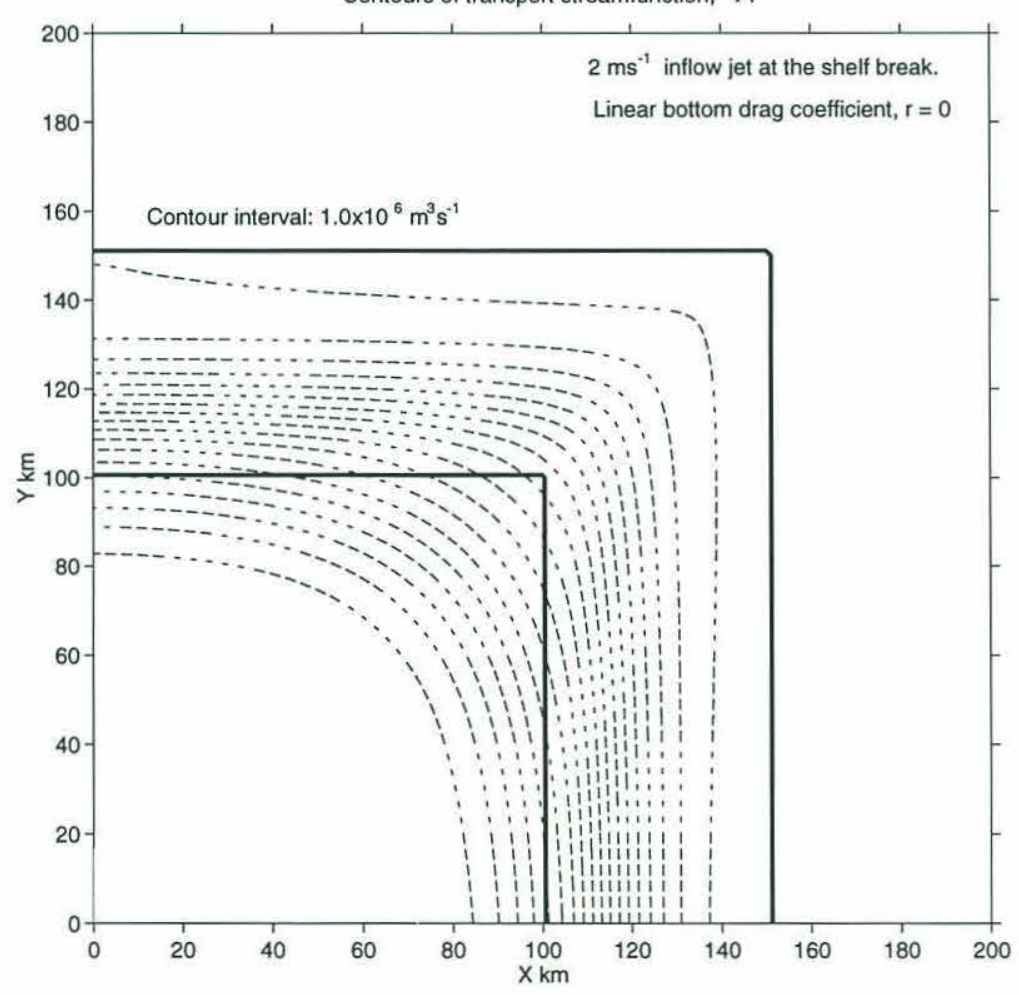

Figure 4.2: Contours of the transport streamfunction for the base case with varying inflow speeds (a) $1.0 \mathrm{~m} \mathrm{~s}^{-1}$ (b) $2.0 \mathrm{~m} \mathrm{~s}^{-1}$. 
The simple model considered in the previous chapter agrees fairly well with the base case on applying the model to several streamlines from different positions across the inflow jet. The Rossby number used in each case was $u_{0} / f L$ where $u_{0}$ is the inflow velocity at that point in the jet and $L=h_{0} / b$, the distance from that point to where the local gradient in the bathymetry would surface if continued upward. This suggests that the adjustment of the jet to the corner in the bathymetry is independent of $\alpha$. To test this, the base case configuration was redone at inflow velocities of 0.1 , 0.2 , and $0.4 \mathrm{~m} \mathrm{~s}^{-1}$ with the inflow jet half as wide. The minimum radius of curvature of the jet at the corner compares well with that for the base case so the dependence on $\alpha$ is weak and will not be considered here.

If the scaling of the flow is independent of $\alpha$, then the scalings obtained from the simple model for the flow at the corner should apply. These are

$$
r_{c}=\frac{\sqrt{\epsilon} L}{0.765}, \quad \frac{h_{0}-h_{c}}{h_{0}}=0.765 \sqrt{\epsilon}
$$

where $r_{c}$ is the radius of curvature at the corner, and $h_{c}$ is the depth at the corner. Figure 4.3 shows the scaling for the curvature at the corner for the series of runs described above. The maximum radius of curvature and the Rossby number estimated for the center of the jet are used. The 'best fit' least squares line through the data has a slope of 0.88 and a y-intercept of -0.0068 . The data lies close to this line and the $y$-intercept is close to zero so this scaling argument seems appropriate for this range of parameters. The slope of the line is less than that predicted by the simple model presumably because the simple model assumes all the negative relative vorticity gained at the corner goes into the turning of the flow and this is not exactly the case for the numerical model results. 


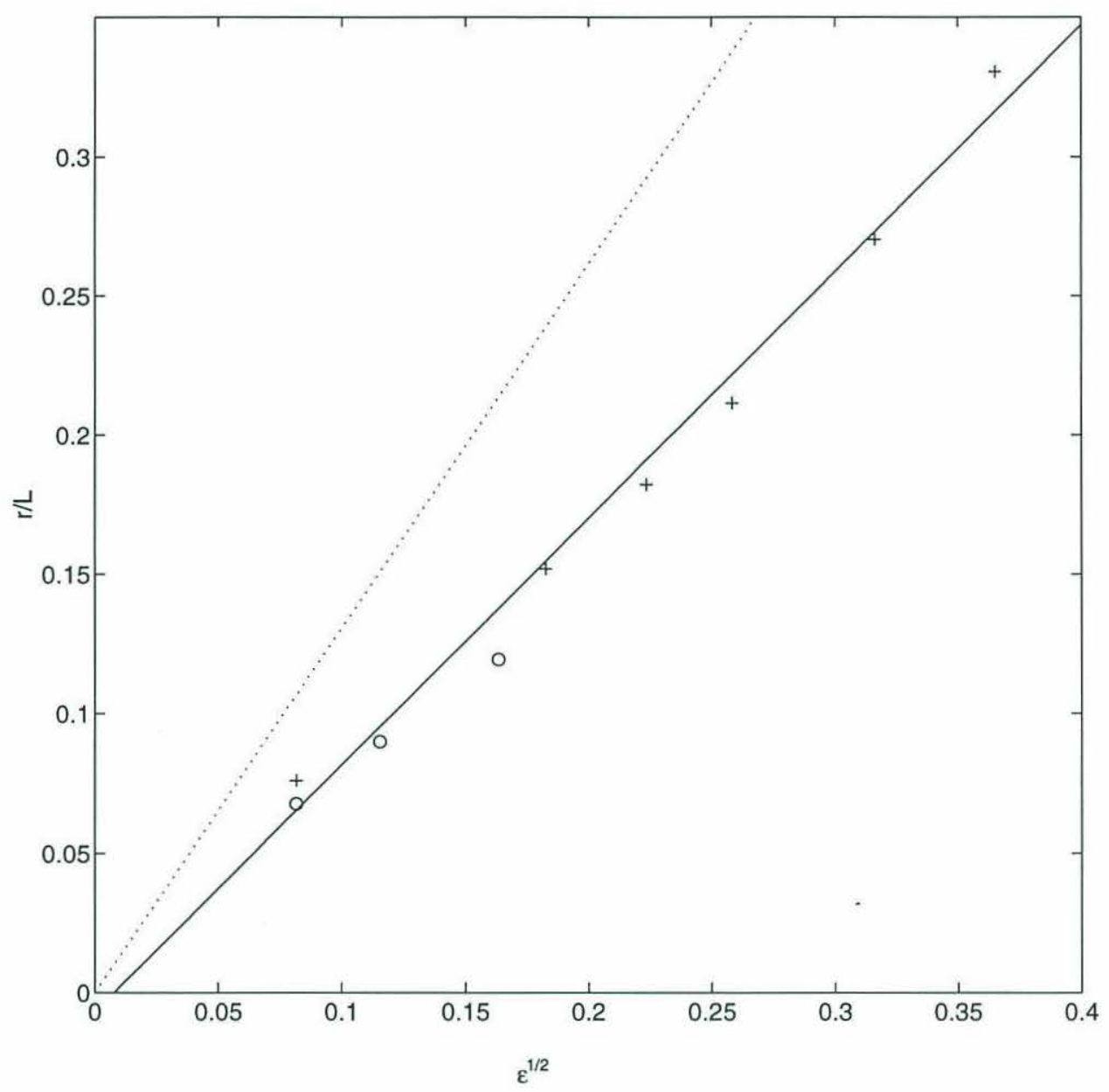

Figure 4.3: $r_{c} / L$ plotted against $\epsilon^{\frac{1}{2}}$. The data are taken from the center of the jet for model runs with inflow velocities of $0.1,0.5,0.75,1.0,1.5$, and $2.0 \mathrm{~m} \mathrm{~s}^{-1}$ for the base case jet (+) and inflow velocities of $0.1,0.2$, and $0.4 \mathrm{~m} \mathrm{~s}^{-1}$ for an inflow jet half as wide (o). The solid line is the 'best fit' line to the data in the least squares sense and the dotted line is the scaling that the simple model would give. 


\subsection{Other Variations}

The linear bottom friction parameterization given in Chapter 2 was used to illustrate the effects of adding bottom friction to the base case. The vertical viscosity used was $0.002 \mathrm{~m}^{2} \mathrm{~s}^{-1}$ which gives an e-folding scale for the Ekman layer of $6.32 \mathrm{~m}$. The linear bottom friction parameter $r_{f}$ was set to $5 \times 10^{-4} \mathrm{~ms}^{-1}$, which gives an Ekman layer with $75 \%$ of the Ekman suction and $59 \%$ of the Ekman transport of an Ekman layer with no-slip bottom friction.

Figure 4.4 (a) shows the flow for the base case configuration with the bottom friction described above. The nature of the adjustment is the same and the change in the flow very slight. The Ekman transport in the bottom boundary is directed off the shelf so the streamlines are slowly deepening where in the base case the flow was parallel to the bathymetry.

All the following runs have similar bottom friction since the decay time of transients during the model spin-up period is much shorter. The effect of friction is always to give a small flow off the shelf, not to radically change the adjustment.

The base case was re-run with a $10-k m$ radius corner instead of a sharp corner (see Figure $4.4(\mathrm{~b})$ ). This gives the inertial radius of the $1.0-\mathrm{m} \mathrm{s}^{-1}$ inflow jet at the shelf-break to be equal to the radius of curvature of the corner. The flow is essentially unchanged by this smoothing of the corner, indicating that an inertial radius criterion does not govern the adjustment of this flow and the scaling presented earlier still applies.

The previous runs all have an inflow jet over the shelf-break. This was chosen so that the flow decayed over the slope and decayed towards the coast and was also stable. The flows over the Scotian Shelf are not necessarily focused at the shelf-break like this. It is likely that the southward flow over the Scotian Shelf is much broader in nature, extending from near the shelf-break to the coast. 
(a)

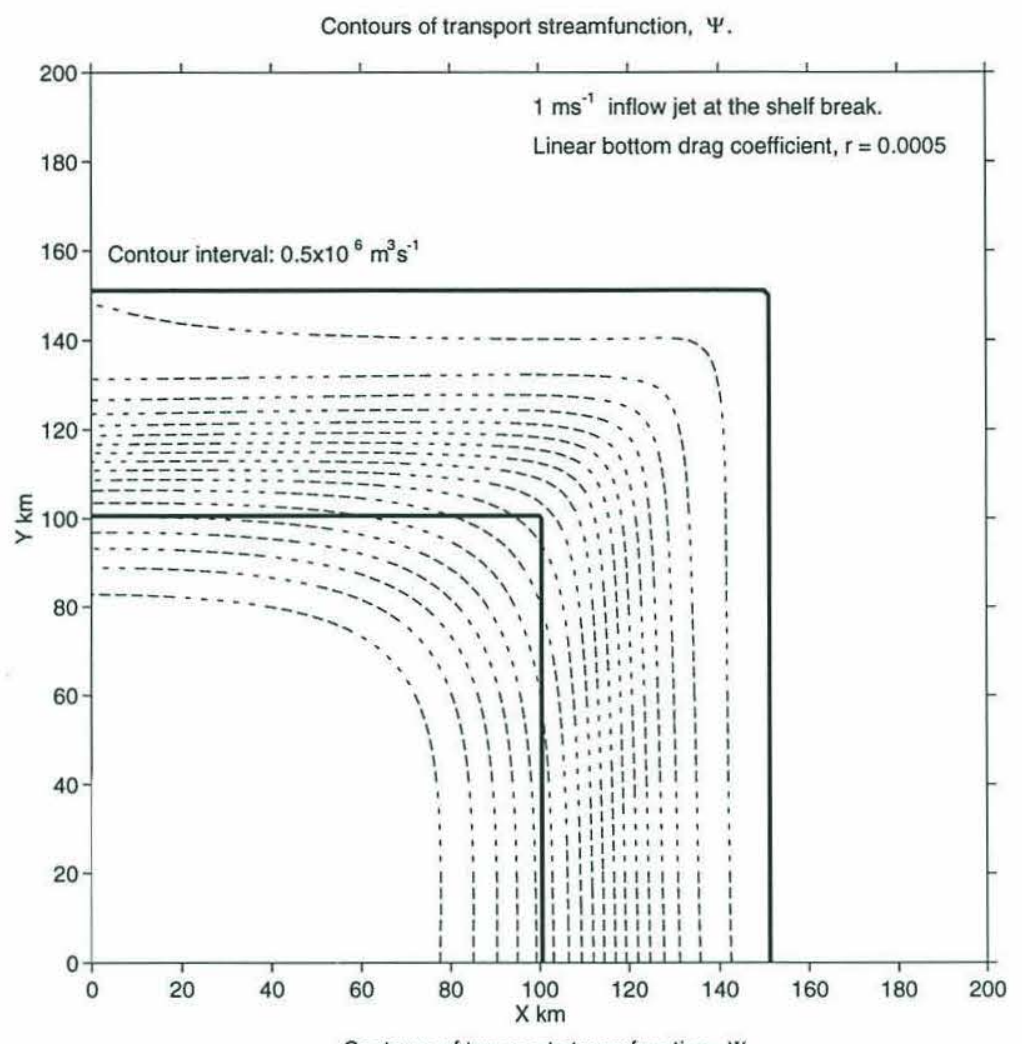

(b)

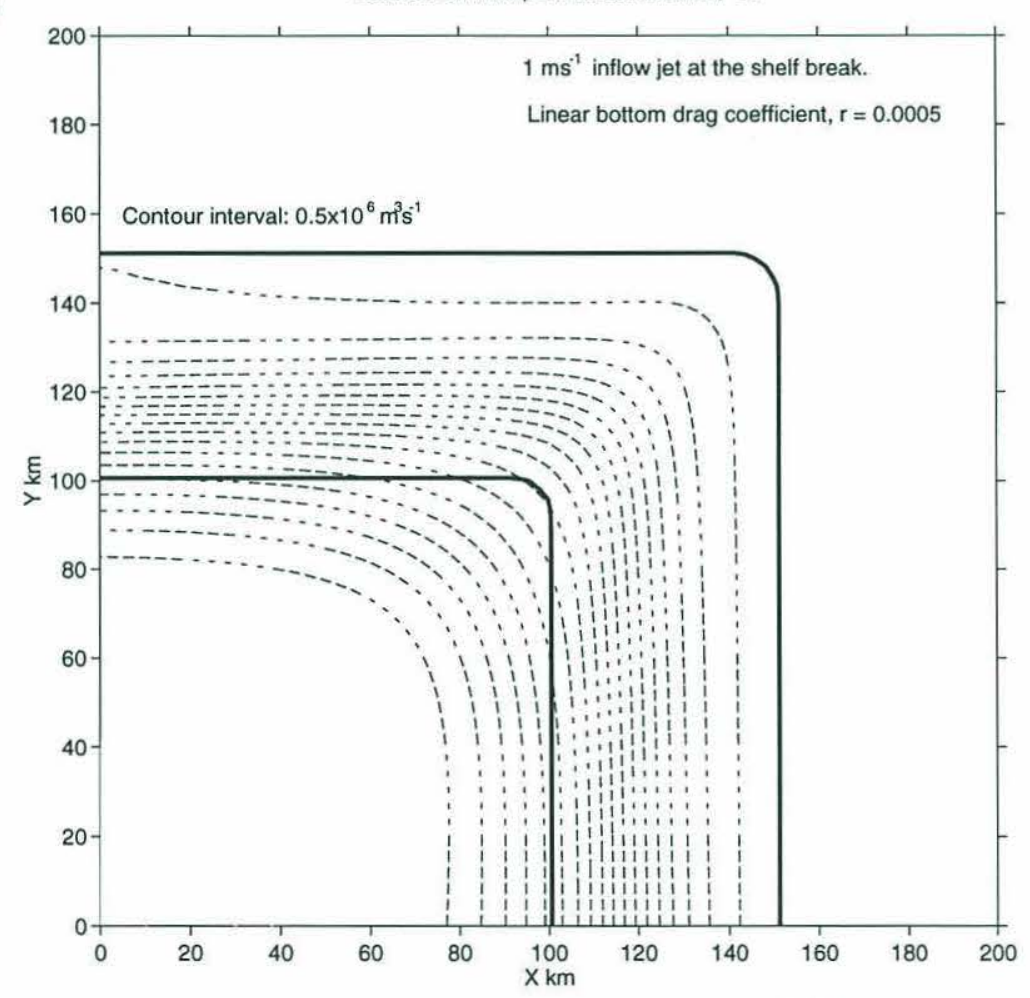

Figure 4.4: Contours of the transport streamfunction for (a) base case with bottom friction,(b) base case with bottom friction and a rounded corner of radius $10 \mathrm{~km}$. 


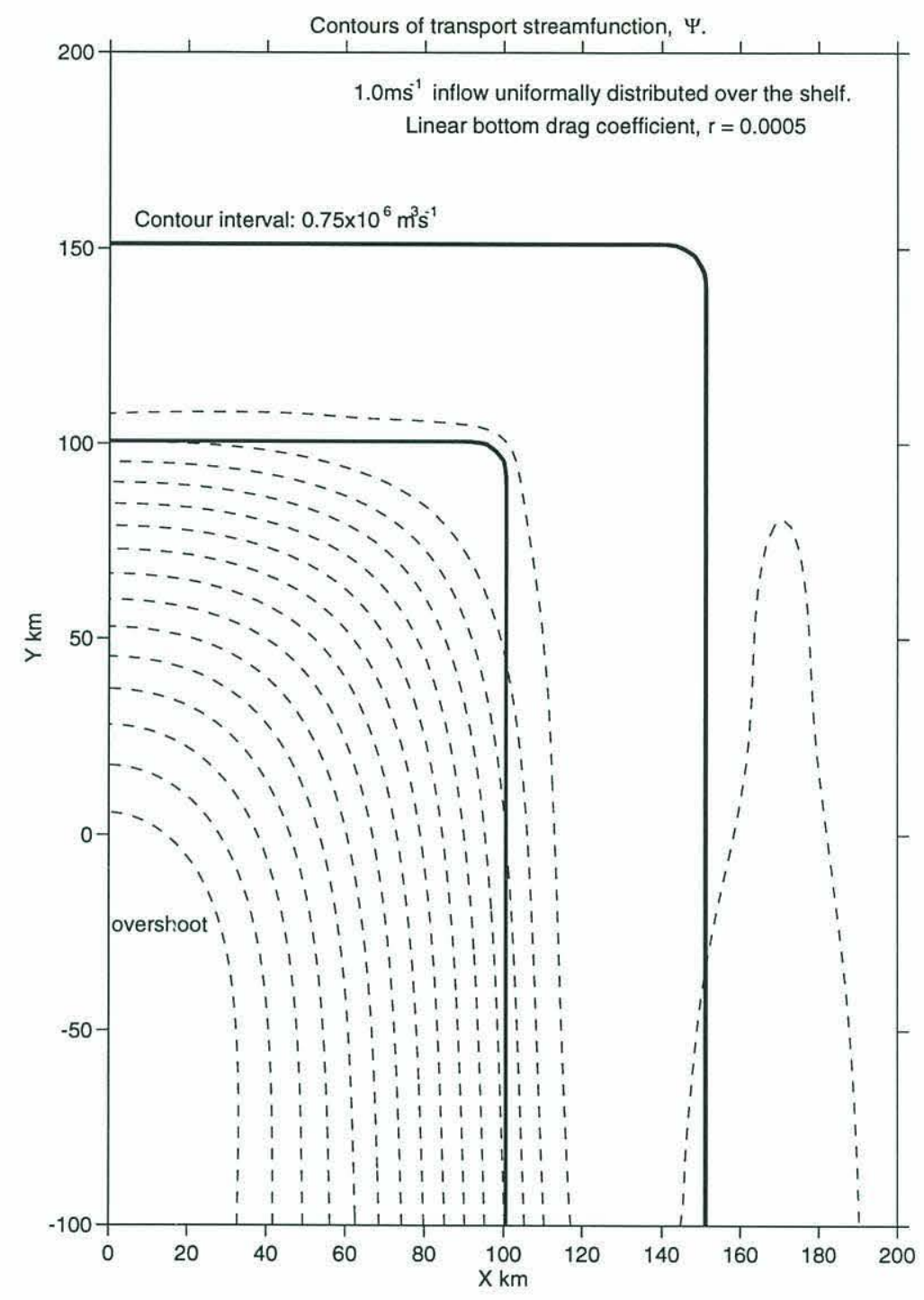

Figure 4.5: Contours of the transport streamfunction for an inflow of $1 \mathrm{~m} \mathrm{~s}^{-1}$ over the shelf. 
Figure 4.5 shows the flow field for a $1 \mathrm{~m} \mathrm{~s}^{-1}$ inflow uniformly distributed over the shelf. The flow turns the corner in a similar manner to the base case. It is necessarily slightly asymmetrically about the corner since at $(0,0) \mathrm{km}$ there is an imposed velocity of $(1,0) \mathrm{m} \mathrm{s}^{-1}$ at the coast. Immediately downstream of the corner in the coastline there is an adjustment region near the coast that appears similar to the results of Klinger (1994). The result of this appears to be to force some fluid off the shelf downstream of the corner.

\subsection{Different Topographies}

Some runs were made with the bottom topography twice as steep and half as wide on the downstream side of the corner. This was done to mimic the Scotian Shelf which is narrower along the inflow side to the Gulf of Maine and also has very steep bathymetry dropping down to the Northeast Channel. The qualitative adjustment at the corner is the same but the flow is no longer symmetrical about the corner since the bottom topography is not symmetrical about the corner. The volume over the shelf is halved in going from the inflow to the outflow and this causes fluid to move off the shelf downstream of the corner.

Also examined was a step rather than a single corner in the shelf-break, which is an abrupt downstream narrowing of the shelf with no overall turn in the shelf-break. Flow adjusts on the scale of the step in this case. The adjustment to the convex part of the step is similar to that found in the base case. However, the flow is not flowing parallel to the step on leaving the corner but is flowing across the bathymetry and adjusting to the concave corner at the downstream end of the step in exactly the opposite manner to the adjustment at the convex corner. This adjustment is not considered important to the dynamics of the separation from the Scotian Shelf since there is no direct continuation of the Scotian Shelf to Georges Bank. 


\section{Chapter 5}

\section{Conclusions}

\subsection{Discussion and Conclusions}

The parameter variations in Chapter 4 indicate that the nature of the adjustment to the sharp bend in the bathymetry that was described in detail in Chapter 3 is robust. For parameters in the oceanographic range, barotropic flow over the shelf/shelf-break adjusts to a $90^{\circ}$ right-hand bend in the shelf bathymetry in a smooth, steady manner. Eddies do not form at the corner as was initially expected. The flow is nearly symmetrical about the corner and it shoals as it approaches the corner and deepens as it leaves, returning to close to its initial depth.

The conservation of shallow water potential vorticity demands that as the flow shoals it gains negative relative vorticity, and as it deepens it loses negative relative vorticity. The comparison between the base case and the simple model in Chapter 3 supports the argument that the gain in negative relative vorticity due to the shoaling at the corner is largely manifested in the turning of the flow. This is how the vorticity adjustment at the corner is consistent with the broad scale turning of the flow around the corner. 
The scaling presented and verified in Chapter 4 demonstrates that the radius of curvature of the flow scales with the square root of the Rossby number $(r \sim \sqrt{\epsilon} L)$. This implies that the adjustment width of the flow becomes broader as the Rossby number increases, and this can be seen in the model results. It is the broad shelf which allows for this broad-scale adjustment of the flow, and means that the local Rossby number of the flow is less than one at the corner. The experiments here are fundamentally different from those of Klinger (1994) where a broad-scale adjustment is not possible because of the presence of the coast and the local Rossby number is greater than one in the adjustment region.

For the model runs presented here, the flows are attached to the bathymetry at the corner rather than overshooting and separating from the corner. Therefore, steady barotropic dynamics and bottom friction do not appear to explain the flow of Scotian Shelf water directly across the Northeast Channel. The initial time-dependent adjustment at the beginning of any model run includes eddies and coastally trapped vorticity waves that are generated at the corner and then propagate downstream. It may be that some kind of overshoot is possible at the corner with time-dependent upstream forcing.

The barotropic model is limited in its applicability to the Scotian Shelf because the density front at the shelf-break of the Scotian Shelf is expected to be dynamically significant. The density front weakens the topographic constraint presented by the Northeast Channel. Having understood the barotropic case, the next step is to include a shelf/slope front in the model to mimic the density stratification found at the shelfbreak of the Scotian Shelf. Both time-dependent forcing and wind-stress could later be put into this baroclinic model as appropriate.

The irreversibility of the vorticity adjustment found in this model suggests further barotropic runs for current impinging on a left-hand bend in the bathymetry where it is expected to flow to the corner and separate. This may have applications to the separation of the Gulf Stream from Cape Hatteras. 


\section{Appendix A}

\section{Stability of a Barotropic Gaussian Jet Over Sloping Bathymetry}

For the inviscid shallow water equations with a rigid lid, the relevant criterion to ensure that a jet flowing parallel to straight bathymetry is stable to small perturbations is that the initial potential vorticity gradient across the jet does not change sign. For a Gaussian jet of the form $u=u_{\max } e^{-\left(\frac{y-L}{w}\right)^{2}}$ flowing along straight bathymetry with a constant bottom slope $b$, and the coast on the right, this criterion implies the jet is stable for

$$
\frac{\alpha^{2}}{\epsilon}>2,
$$

where $\alpha=W / L, \epsilon=u_{\max } / f L$ and $L$ is the distance to the coast as in the simple model. Note that $\alpha^{2} / \epsilon \propto 1 / L$ so that as $L$ decreases the jet bcomes more stable for a given $u_{\max }$ and $W$. The jet broadens as $\alpha$ increases and comes into contact with the coast for $\alpha>0.5$, so we cannot consider very broad jets in the numerical model independently of the boundary conditions at the coast, and the range of Rossby numbers is restricted to $0<\epsilon<0.1$.

The shelf/slope topography used in the numerical model stabilizes the inflow jet in the base case. If the slope is continued upward it reaches the surface very close 
to the center of the jet so that for the left-hand side of the jet, which is over the slope, $L$ is small and $\alpha^{2} / \epsilon>2$ for the range of $\epsilon$ and $\alpha$ used in this study. The right-hand side of the jet is over the shelf where $L$ is large and $\alpha^{2} / \epsilon<2$. The jet as a whole is found to be stable. 


\section{Bibliography}

Bisagni, J. J., R. C. Beardsley, C. M. Ruhsam, J. P. Manning, and W. J. Williams. Historical and Recent Evidence Concerning the Presence of Scotian Shelf Water on Southern Georges Bank. Deep-Sea Research. submitted.

Camerlengo, A. L. and J. O'Brien, 1980. Open Boundary Conditions in Rotating Fluids. J. Computational Physics, 35, 12-35.

Chapman, D. C., 1985. Numerical Treatment of Cross-Shelf Open Boundaries in a Barotropic Coastal Ocean Model. J. Physical Oceanography, 15, 1060-1075.

Chapman, D. C., J. A. Barth, R. C. Beardsley, and R. G. Fairbanks, 1986. On the Continuity of the Mean Flow between the Scotian Shelf and the Middle Atlantic Bight. J. Physical Oceanography, 16(4), 758-772.

Flagg, C. N., 1987. Hydrographic Structure and Variability. Georges Bank, R. Backus, Ed., MIT Press, 108-124.

Gawarkiewicz, G. and D. C. Chapman, 1991. Formation and Maintenance of Shelfbreak Fronts in Unstratified Flow. J. Physical Oceanography, 21(8), 1225-1239.

Gawarkiewicz, G. and D. C. Chapman, 1995. A numerical study of dense water formation and transport on a shallow, sloping continental shelf. J. Geophysical Research, 100(C3), 4489-4507.

Haidvogel, D. B., J. L. Wilkin, and R. Young, 1991. A semi-spectral Primitive Equation Ocean Model Using Vertical Sigma and Orthogonal Cuvilinear Horizontal Coordinates. J. Computational Physics, 94(1), 151-185.

Klinger, B. A., 1993. Gyre formation at a corner by rotating barotropic coastal flows along a slope. Dynamics of Atmospheres and Oceans, 19, 27-63.

Klinger, B. A., 1994. Baroclinic eddy generation at a sharp corner in a rotating system. J. Geophysical Research, 99(C6), 12,515-12,531.

Klinger, B. A., 1994. Inviscid Current Separation from Rounded Capes. J. Physical Oceanography, 24(8), 1805-1811.

Mesinger, F. and A. Arakawa, 1976. Numerical Methods Used in Atmospheric Models, volume 1. GARP. 
Orlanski, I., 1976. A Simple Boundary Condition for Unbounded Hyperbolic Flows. J. Computational Physics, 21, 251-269.

Robinson, R. A. and P. P. Niiler, 1967. The theory of free inertial currents. Tellus, XIX(2), 269-291.

Schwing, F. B., 1992. Subtidal Response of Scotian Shelf Circulation to Local and Remote Forcing. Part 1: Observations. J. Physical Oceanography, 22, 523-541.

Smith, P. C., 1983. The Mean and Seaonal Circulation off Southwest Nova Scotia. J. Physical Oceanogaphy, 13(6), 1034-1054.

Smith, P. C. and B. D. Petrie, 1982. Low-Frequency Circulation at the Edge of the Scotian Shelf. J. Physical Oceanography, 12, 28-46. 